\title{
Laser-Induced Fluorescence of Singly-Charged Xenon inside a 6-kW Hall Thruster
}

\author{
Wensheng Huang ${ }^{*}$, Brittany Drenkow ${ }^{\dagger}$, and Alec D. Gallimore \\ Plasmadynamics and Electric Propulsion Laboratory, University of Michigan, Ann Arbor, MI 48109
}

\begin{abstract}
We present laser-induced fluorescence velocimetry measurements of the $5 \mathrm{~d}[4]_{7 / 2} \rightarrow$ $6 \mathrm{p}[3]^{\circ}{ }_{5 / 2}$ transition for singly-charged xenon (Xe II) at $834.953 \mathrm{~nm}$ (vacuum) inside and in the near-field plume of a 6-kW Hall thruster. The thruster has a nominal operating discharge voltage of $300 \mathrm{~V}$ and a nominal anode mass flow rate of $20 \mathrm{mg} / \mathrm{s}$. Axial velocity profiles are obtained along the centerline of the discharge channel. The thruster is operated under seven conditions spanning discharge voltages of 150-600 V and anode mass flow rates of 10-30 $\mathrm{mg} / \mathrm{s}$. The near-field results match those obtained in a previous experiment on the same thruster, validating the new optical fiber setup. The lengths of the acceleration zone are found to vary from 14 to $26 \mathrm{~mm}$. The location of the acceleration zone appears to move downstream as the flow rate increases but moves upstream as the discharge voltage increases. The signal to noise ratio varies greatly and is generally an order of magnitude lower at locations believed to be inside the Hall current. At these locations, the widths of the velocity distributions are several times wider than those found elsewhere and the distributions are often bimodal. This phenomenon is likely to be the result of taking timeaveraged measurements on a highly oscillatory plasma. By deconvolving the pre-accelerated velocity distribution function from the downstream distributions, we can roughly approximate the minimum and maximum instantaneous bulk velocities found at each scan location. The plot of these velocities supports the possibility that the entire acceleration zone is oscillating back and forth in space.
\end{abstract}

\section{Introduction}

$\mathrm{A}$ S Hall thruster technology begins to mature in the United States, more and more attention is being paid toward predicting thruster performance and lifetime. However, modeling efforts have suffered due to a lack of understanding about the fundamental physical principles behind phenomena found inside the Hall thruster such as plasma oscillations, which will be the main focus of this paper. In particular, characterization of the breathing mode may be vital toward predicting wall erosion and understanding cross-field electron transport.

The oscillatory nature of the plasma properties in the plume of a Hall thruster has been repeatedly observed in many experiments. ${ }^{1-5}$ Great theoretical advances have been made in understanding and explaining these oscillations. Yet, there is a lack of non-intrusive experimental data characterizing the behavior of plasma oscillations inside the Hall thruster, from where the oscillations in the plume originate.

Laser induced fluorescence (LIF) is an optical technique that can extract non-intrusive, species-specific, and spatially-resolved information from inside the discharge channel of a Hall thruster. While traditionally used to obtain time-averaged velocity distribution functions (VDF) in the Hall thruster community, ${ }^{6-10}$ this paper will demonstrate the possibility of obtaining probabilistic transient information about Hall thruster oscillations.

The goal of this paper is to summarize the findings of a LIF experiment to characterize the behavior of singlycharged xenon ions inside of the discharge channel of a $6-\mathrm{kW}$ Hall thruster. We used the Xe II $5 \mathrm{~d}[4]_{7 / 2} \rightarrow 6 \mathrm{p}[3]^{\circ}{ }_{5 / 2}$ transition at $834.953 \mathrm{~nm}$ (vacuum) to excite the xenon ions and collected non-resonant fluorescence at $541.9 \mathrm{~nm}$ (air). Similar LIF experiments have been performed by Hargus, et al., on lower power thrusters. ${ }^{6-8}$ Due to the lack of published hyperfine structure constants for the Xe II $834.953 \mathrm{~nm}$ (vacuum) transition, we used a method involving direct deconvolution of an experimentally obtained hyperfine structure lineshape from the raw LIF data. The method is described in a previous paper. ${ }^{11}$ The results of the experiment described in this paper show regions in the

\footnotetext{
*Ph. D. Candidate, Aerospace Engineering, davhuang@umich.edu, Student Member, AIAA.

† Undergraduate, Aerospace Engineering, bdrenkow@umich.edu, Student Member, AIAA.

* Arthur F. Thurnau Professor, Aerospace Engineering, alec.gallimore@umich.edu, Associate Fellow, AIAA.
} 
acceleration zone where the full-width-at-half-maximum (FWHM) of the VDF is found to be a few times larger than anywhere else. It is likely that the extra wide VDF results from performing time-averaged LIF on a highly oscillatory plume; other causes are also discussed.

To obtain probabilistic transient information, a statistical method is used to extract the spatial behavior of the instantaneous bulk velocity. This method involves deconvolving the VDFs obtained in the accelerating plasma plume by a VDF found upstream of the acceleration zone. The results of the method is expected to give only a rough estimate of the plasma oscillation in space as a number of lesser broadening effects will be ignored. However, this result can be looked at qualitatively to gain a better understanding of how the plasma oscillates inside the 6-kW Hall thruster. The various assumptions used in the described method and the validity of these assumptions will then be addressed.

\section{Background Theories}

Sections A and B lay out the theory underlying the extraction of time-averaged VDFs from raw LIF spectra. Section $\mathrm{C}$ breaks down the data components that make up a time-averaged VDF thus obtained. This section lays the theoretical foundation for the extraction of probabilistic transient information. Section D briefly describes existing theories regarding breathing mode, Hall thruster plasma oscillations, and their relationship to the LIF signal-to-noise ratio. For the remainder of this paper, all transition wavelengths are given in vacuum values and all intensities given in arbitrary units, a.u., unless otherwise specified. Note that any discussion of temperature is generally avoided as the plasma under study is not expected to be in thermal equilibrium given the discharge channel plasma conditions. For the most parts, full-width-at-half-maximum (FWHM) values are discussed instead.

\section{A. Principles of Laser-Induced Fluorescence Velocimetry}

LIF operates on the principle that a particle (an atom or molecule) absorbs a photon at a shifted frequency when moving due to the Doppler Effect. The particle then spontaneously emits a photon when it de-excites. This spontaneous emission, called fluorescence, radiates isotropically away from the particle. For a particle travelling at non-relativistic speed, the shift in absorption frequency is proportional to the particle velocity component in the direction that the photon travels. The mathematical relation is given by Eq. (1),

$$
\frac{\Delta v}{v_{0}}=-\frac{\mathbf{v}}{\mathrm{c}} \cdot \frac{\mathbf{k}}{|\mathbf{k}|}
$$

where $v_{0}$ is the photon frequency, $\Delta v$ is the shift in photon frequency from the perspective of the particle, $\mathbf{v}$ is the particle velocity, $\mathbf{c}$ is the speed of light, and $\mathbf{k}$ is the photon wave vector. By varying the frequency of the injected photons and comparing the intensity of the collected fluorescence, we can obtain the particle VDF along the injected photon wave vector. In this experiment, light is injected at around $835 \mathrm{~nm}$ and non-resonant LIF is collected at $542.066 \mathrm{~nm}$. Figure 1 shows the transition diagram. Due to the nature of LIF, this experiment actually measures the VDF of the excited state $5 \mathrm{~d}[4]_{7 / 2}$, which is assumed to have the same VDF as the entire ion population.

\section{B. Hyperfine Structure and Deconvolution}

Analysis of LIF data is complicated by the presence of broadening factors, which can distort and widen the raw data. For the Xe II $5 \mathrm{~d}[4]_{7 / 2} \rightarrow 6 \mathrm{p}[3]^{\circ}{ }_{5 / 2}$ transition, a prominent broadening factor is the hyperfine structure. The hyperfine structure constants for the lower state of the Xe II $834.953 \mathrm{~nm}$ transition are not known. However, the hyperfine structure has been shown to be around $500 \mathrm{MHz}$ wide in photon frequency space. ${ }^{11}$ The FWHM of the average ion VDF in a Hall thruster typically varies from 1 to $10 \mathrm{~km} / \mathrm{s}$, which for this transition corresponds to about 1.2 to $12 \mathrm{GHz}$. For the parts of the plasma where the velocity distributions are wide, the hyperfine structure will present a negligible broadening of the lineshape. But for those parts with narrow distributions, like the plasma upstream of the acceleration zone, removal of hyperfine effect is important. Natural (or lifetime) broadening, which is due to the Heisenberg uncertainty principle, is also present. This effect broadens an absorption line into a Lorentzian function. The natural broadening effect is

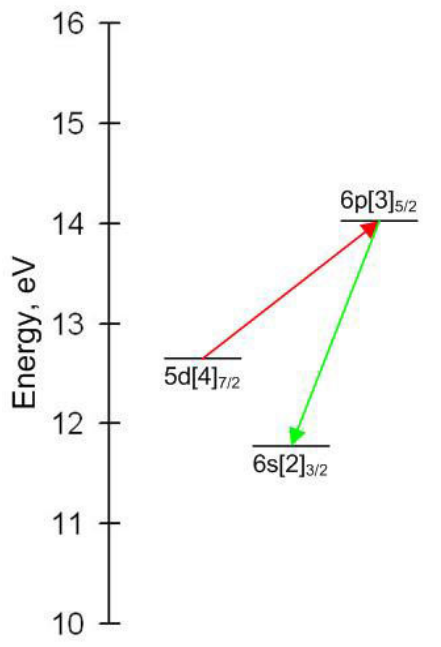

Figure 1. Transition diagram for Xe II LIF at $834.953 \mathrm{~nm}$. Wavelength in vacuum. 
believed to be negligible compared to the hyperfine structure effect based on measurements at other xenon ion lines. Specifics of hyperfine structure and natural broadening theories can be found in Svanberg. ${ }^{12}$

Furthermore, external electric and magnetic fields can increase the number of hyperfine splittings. For the purpose of this experiment, we will try to avoid these distortions as much as possible. These externally influenced splittings depend on the direction and polarity of the injected laser beam. The electric field present in a typical Hall thruster is several orders of magnitude smaller than what is necessary to cause distortions, called the Stark effect. ${ }^{13}$ The magnetic field effect for weak field strengths $(\sim 100 \mathrm{G})$, called the Zeeman Effect, can be categorized under $\sigma$ and $\pi$-polarization. The beam is $\sigma$-polarized if the polarization of the beam is perpendicular to the external magnetic field $(\mathbf{E} \perp \mathbf{B})$. The beam is $\pi$-polarized if the polarization of the beam is parallel to the external magnetic field ( $\mathbf{E} \|$ B). For the Xe II $5 \mathrm{~d}[4]_{7 / 2} \rightarrow 6 \mathrm{p}[3]_{5 / 2}^{0}$ transition, $\pi$-polarized splitting effects are negligible while $\sigma$-polarized splitting effects are not. ${ }^{14}$ By injecting the laser beam with radial polarization to match the approximately radial magnetic field at the Hall thruster exit plane, we can obtain LIF scans with negligible Zeeman splitting.

Deconvolution is a mathematical process by which broadening effects can be removed. Due to the lack of knowledge regarding the hyperfine structure constants for the transition used, past LIF experiments have either used the most probable velocity to characterize the results ${ }^{15}$ or did not deconvolve the hyperfine structure out of the result and accepted higher uncertainty ${ }^{10}$. In a previous study, a slightly Doppler-broadened hyperfine structure lineshape was experimentally obtained via intermodulated optogalvanic spectroscopy. This lineshape is used as the basis for deconvolution, thereby bypassing the need to know the hyperfine structure constants. The methodology and validity for this approach is discussed in a previous paper. ${ }^{11}$

\section{Components of Time-Averaged Laser-Induced Fluorescence Data}

Time-averaged LIF data are mathematically represented as a convolution of a number of different components. Equation (2) shows a typical breakdown. Note the domain is written in photon frequency, which has a one-to-one correspondence with particle velocity along the laser axis.

$$
\overline{\mathrm{i}}(\mathrm{v})=(\mathrm{h}(\mathrm{v}) \otimes \operatorname{nat}(\mathrm{v})) \otimes \overline{\mathrm{g}}(\mathrm{v})
$$

In this equation, $\overline{\mathrm{i}}(\mathrm{v})$ is the raw lineshape obtained from time-averaged LIF, $\mathrm{h}(\mathrm{v})$ is the hyperfine structure lineshape, nat $(v)$ is the natural broadening lineshape, and $\bar{g}(v)$ is the time-averaged VDF in frequency space. The $\otimes$ symbol represents the mathematical process called convolution, which is defined in Eq. (3)

$$
\mathrm{C}(\mathrm{x})=\int_{-\infty}^{\infty} \mathrm{A}(\mathrm{x}-\mathrm{y}) \mathrm{B}(\mathrm{y}) \mathrm{dy}
$$

where the function $\mathrm{C}$ is the result of the convolution and the function $\mathrm{A}$ is said to be convolved with the function $\mathrm{B}$. Note deconvolution is the inverse of convolution. By using the direct deconvolution method referenced in the previous section, we are effectively removing $h(v)$ and nat $(v)$ from the raw lineshape.

We can now break down the time-averaged VDF, $\bar{g}(v)$, into time-dependent components as follows,

$$
\bar{g}(v)=\frac{1}{\tau} \int_{0}^{\tau} \int_{-\infty}^{\infty} f(v-\hat{v}, t) p d f(\hat{v}, t) d \hat{v} d t, \tau>\tau_{\text {osc }}
$$

where $\mathrm{f}$ is the instantaneous stationary VDF, $\hat{v}$ is the instantaneous bulk velocity, $\tau_{\text {osc }}$ is the plasma oscillation period, and pdf is the probability density function (PDF) of the instantaneous bulk velocity. The instantaneous stationary VDF, $\mathrm{f}$, is defined as the instantaneous VDF with the velocity axis shifted by the instantaneous bulk velocity, such that the ensemble-averaged velocity of $f$ is always 0 . Note that probability density function, PDF, is not to be confused with velocity distribution function, VDF. The instantaneous bulk velocity PDF is a function that describes the probability with which the instantaneous bulk velocity will take on a particular value at a given time. We also need to make a distinction between time-averaged bulk velocity, which will be simply referred to as the bulk velocity, and the instantaneous bulk velocity. The bulk velocity is the velocities of all of the particles averaged over a period of time much longer than the plasma oscillation periods. The instantaneous bulk velocity is the ensemble-averaged velocity of all of the particles at a particular moment in time. Let us define a quasi-steady plasma as a plasma whose properties do not change over all time scales much longer than the plasma oscillation periods. For such a plasma, the pdf term in Eq. 4 becomes independent of time and $\tau$ approaches infinity. 
Figure 2 below shows an example of the concept described in Eq. 4. Note that all subplots in Fig. 2 are plotted against axial velocity even though the primary dependent variable in Eq. 4 is photon frequency because axial velocity has a one-to-one correspondence with photon frequency for LIF velocimetry. In this example, the plasma is quasi-steady with an instantaneous stationary VDF that does not vary in time. However, the instantaneous bulk velocity undergoes sinusoidal oscillation over time due to some perturbing plasma oscillation. Figure 2a illustrates the evolution of the instantaneous bulk velocity of this hypothetical plasma. Figure $2 \mathrm{~b}$ shows the probability density function (PDF) describing the likelihood that the instantaneous bulk velocity will take on a particular value at a given time, and represents the pdf term in Eq. 4. Figure $2 \mathrm{c}$ shows the instantaneous stationary velocity distribution function (VDF) of the hypothetical plasma, which corresponds to the $\mathrm{f}$ term in Eq. 4. Since there is no time dependence, we can plot $\mathrm{f}$ against only the velocity in this example. Figure $2 \mathrm{~d}$ shows the time-averaged LIF results that one would obtain when measuring the said hypothetical plasma. These four subplots together illustrate how an oscillating plasma can produce an apparently bi-modal time-averaged LIF trace. These subplots also illustrate the difference between the instantaneous stationary VDF component (Fig. 2c) and the instantaneous bulk velocity PDF component (Fig. 2b) of a time-averaged LIF trace. It is interesting to note that for this hypothetical plasma Fig. 2d can be obtained by simply convolving Fig. $2 b$ with Fig. $2 c$ as illustrated by the $\otimes$ and $=$ symbols in Fig. 2 .
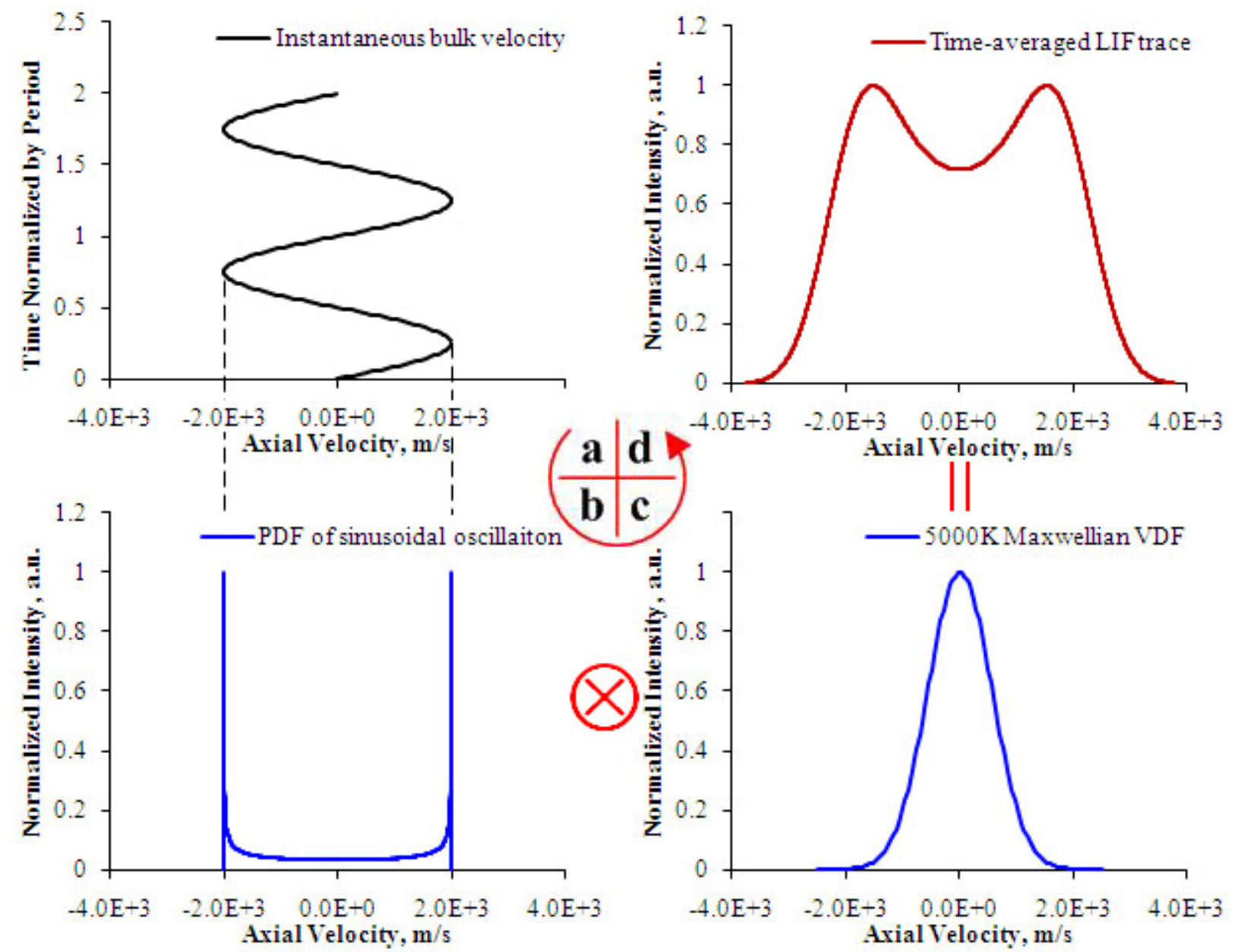

Figure 2. Example illustrating the components of a time-averaged LIF trace obtained from a hypothetical oscillating plasma. Starting from the upper left corner, going counter-clock-wise, the subplots are assigned the letters a, $b$, c, and d. Figure 2a is a plot of the oscillation of the bulk velocity of this hypothetical plasma. Figure 2b is the probability density function (PDF) of the bulk velocity for such a plasma. Figure $2 \mathrm{c}$ is the instantaneous stationary velocity distribution function (VDF) of the hypothetical plasma. And Fig. $2 d$ is the time-averaged LIF trace produced from measuring the said plasma. 


\section{Hall Thruster Plasma Oscillations and the Breathing Mode}

The plasma of a Hall thruster undergoes a large variety of oscillations, many of which have been previously studied. ${ }^{1-5}$ These oscillations typically range from $10 \mathrm{kHz}$ to $10 \mathrm{GHz}$. The resulting plasma potential fluctuations lead to velocity fluctuations, which in general show up as extra broadening in time-averaged VDFs. In terms of Eqn. (4), these broadening factors can show up in both the instantaneous stationary VDF term and the PDF term depending on the nature of the oscillation. This paper will focus mainly on the breathing mode.

The breathing mode is based on the "predator and prey" principle of propellant ionization. Electrons are the predator and neutral xenon are the prey. When an electron ionizes a neutral, an electron-ion pair is born. The ion then sees a high electric field and quickly leaves the ionization zone. The two electrons pick up energy and become trapped in the Hall current until they collide with other neutrals. Thus, the electron population grows as the neutrals are "eaten" up until there are few neutrals left. At this point, the remaining electrons reach upstream of the ionization zone where the potential drop is low and they cannot pick up enough energy to ionize again before reaching the anode. Then, a new wave of electrons is pulled in from the cathode and meets a new wave of neutrals from the anode. The process repeats itself. Detail physical analysis of this phenomenon can be found in Ref. 16 and 17.

\section{Experimental Setup}

\section{A. Facility}

Experiments were performed in the Large Vacuum Test Facility (LVTF) of the Plasmadynamics and Electric Propulsion Laboratory (PEPL) at the University of Michigan. The LVTF is a $\varphi 6 \mathrm{~m} \times 9 \mathrm{~m}$ stainless steel-clad cylindrical chamber. Due to the size of the chamber, the thruster plume is able to expand un-impeded until termination at a beam dump $\sim 4 \mathrm{~m}$ downstream. Pumping is provided by seven cryopumps with a nominal xenon pumping speed of $240,000 \mathrm{l} / \mathrm{s}$. Facility pressure is monitored by two hot-cathode ionization gauges. The base pressure is approximately $4 \times 10^{-7}$ Torr. The pressure during thruster operation at $30 \mathrm{mg} / \mathrm{s}$ anode mass flow rate is approximately $4 \times 10^{-6}$ Torr, corrected for xenon.

During the experiments, the 6-kW Hall thruster was mounted on two cross-mounted stepper-motor translation stages. The stages provide $1 \mathrm{~m}$ of axial travel and $2 \mathrm{~m}$ of radial travel accurate to within $0.01 \mathrm{~mm}$. Laser injection and LIF collection optics were fixed to the floor of the chamber.

Research-grade xenon propellant $(99.999 \%$ pure) was supplied to the thruster by commercially available flow meters and controllers, having an accuracy of $\pm 1 \%$. Calibration of the flow system was done by the constant volume method taking into account the effects of compressibility.

\section{B. Thruster}

The 6-kW annular Hall thruster nominally operates at $300 \mathrm{~V}$ discharge voltage and $20 \mathrm{mg} / \mathrm{s}$ anode mass flow rate. Table 1 lists the operating conditions tested during this experiment. Note that the discharge voltage and anode mass flow rate are the main varying parameters between conditions. For the rest of this paper, operating conditions are labeled as YYY V, ZZ mg/s, where YYY is the discharge voltage and $\mathrm{ZZ}$ is the anode mass flow rate. Cathode mass flow rate is fixed to $7 \%$ of the anode mass flow rate for all operating conditions. Magnetic field settings are chosen to create roughly symmetric magnetic fields (about the thruster channel centerline) and to maximize thruster efficiency. These settings were found through the use of an inverse pendulum thrust stand.
Table 1. List of operating conditions

\begin{tabular}{|c|c|}
\hline $\begin{array}{l}\text { Discharge } \\
\text { voltage, } \mathbf{V}\end{array}$ & $\begin{array}{l}\text { Anode mass } \\
\text { flow rate, } \mathbf{~ m g} / \mathbf{s}\end{array}$ \\
\hline 150 & $\mathbf{1 0}$ \\
\hline 150 & $\mathbf{2 0}$ \\
\hline 150 & $\mathbf{3 0}$ \\
\hline 300 & $\mathbf{1 0}$ \\
\hline 300 & 20 \\
\hline 300 & 30 \\
\hline 600 & 10 \\
\hline
\end{tabular}

\section{Air-Side Laser and Injection Optics}

Figure 3 shows the air-side laser and optics setup for the LIF experiment. The laser system used is a TOPTICA TA-100/830 tapered-amplifier diode laser. This system has a nominal linewidth of $\sim 10 \mathrm{MHz}$ and a mode-hop-free range of $\sim 12 \mathrm{GHz}$. Output power is limited to $\sim 250 \mathrm{~mW}$ at $834.953 \mathrm{~nm}$ (vacuum).

Two relatively weak beams are split off from the main laser beam and sent into the Burleigh SA-91 etalon assembly and the Burleigh WA-1000 wavemeter for reference information. A third relatively weak beam is split off and sent into the optogalvanic cell for stationary reference. This beam is mechanically chopped at $\sim 1.1 \mathrm{kHz}$.

The optogalvanic cell used in this study is a Hamamatsu L2783-42 XeNe-Mo galvatron. The core of the galvatron is a pair of $\varphi 6.25 \mathrm{~mm}$ cylindrical Mo tube electrodes centered in a $\varphi 25 \mathrm{~mm}$ x $120 \mathrm{~mm}$ glass cylinder. The cylinder is filled with approximately 3 Torr of xenon and 4 Torr of neon. The ends of the cylinder are angled at approximately 10 degrees from being perpendicular to the electrode axis to eliminate retro-reflection. The galvatron 


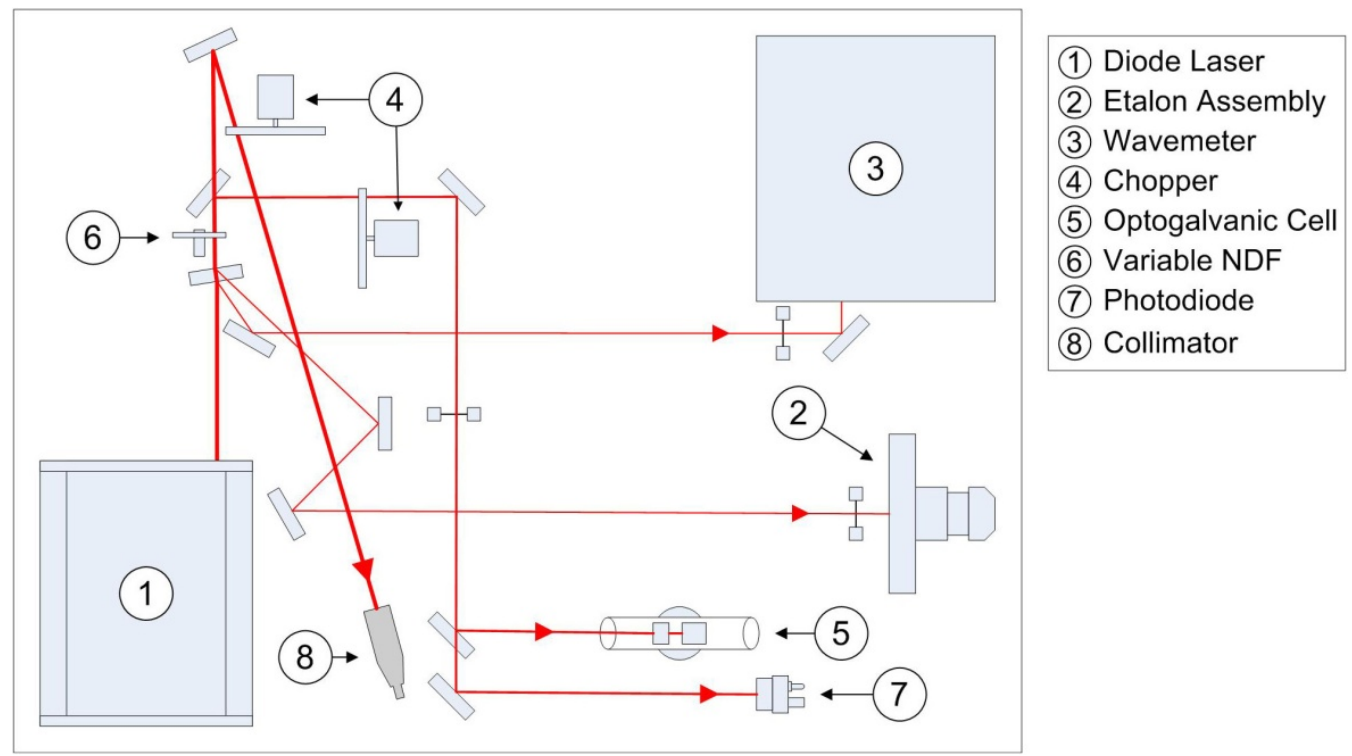

Figure 3. Air side optical setup.

is operated at $250 \mathrm{~V}$ to maintain a warm, dense, and stationary plasma rich with ion species. The voltage drop across the cell's ballast resistor is connected to the SR-810 lock-in amplifier through an RC filter that passes only the AC component of the signal.

A fourth weak beam is split into a Thorlabs DET-110 photodiode to monitor laser power drift. This reading is fed into another SR-810 lock-in amplifier for signal collection. For a relatively unsaturated LIF trace, this laser power reading is used to remove the effects of laser power drift from the trace.

The remaining beam power is mechanically chopped at $\sim 1.8 \mathrm{kHz}$ via an SR540 chopper, and sent into a fiber collimator. The fiber delivers the light through an optical feedthru into the vacuum chamber.

When doing saturation study, an additional variable neutral density filter is added so that the laser power being injected into the chamber can be fine tuned.

\section{Vacuum-Side Optics}

Figure 4 shows a diagram of the vacuum-side optical and thruster setup. The injection optics sends the laser axially into the thruster. This beam is focused down to a point with a $1-\mathrm{mm}$ diameter circular cross section via an anti-reflect-coated plano-convex lens. A polarizer (not shown in diagram) is placed between the optical fiber output and the lens to provide horizontal polarization with respect to the chamber floor. The injection beam focusing cone has a half-angle of $\sim 0.5^{\circ}$ and the injection axis is aligned to within $0.1^{\circ}$ of the thruster firing axis so cosine losses are negligible. The interrogation zone is at the 9 o'clock position when viewing the thruster face on. To prevent excess beam drift during thruster operation a shield optic is installed to allow the laser through while blocking the incoming xenon particles.

The collection optics is built from a matching pair of anti-reflect-coated achromatic lenses. Thermal shielding is not necessary for these optics based on thermocouple measurements taken during the test. Nevertheless, some thermal drift takes place over the course of the test so a reference pin is installed to the left of the thruster when viewing face on. The exact position of this pin relative

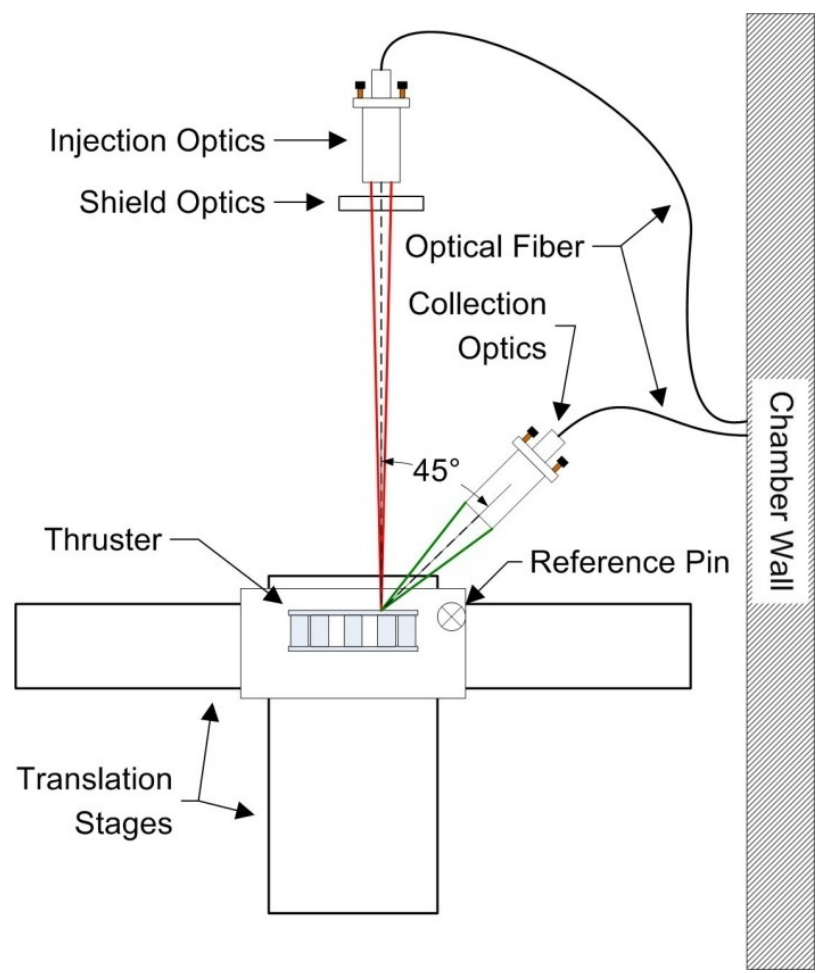

Figure 4. Vacuum side optical and thruster setup. 
to the thruster is measured and the reflected laser signal from this pin is used to compensate for thermal drift. The collection optics is set at a $45 \pm 1$ degree angle with respect to the thruster firing axis to allow the interrogation of the inside of the thruster channel. The resulting intersection of the injection and collection optics forms a diagonally sliced cylinder $\sim 1 \mathrm{~mm}$ in diameter in the radial direction and $\sim 1.5 \mathrm{~mm}$ long in the axial direction. This interrogation zone size sets the spatial resolution of the LIF measurements.

\section{E. Air-Side Collection Optics}

An optical fiber brings the fluorescence signal from the chamber to a SPEX-500M monochromator, the output of which is amplified by a Hamamatsu R928 photomultiplier. The amplified signal is sent to a third SR-810 lock-in amplifier. LIF scans were taken with different slit sizes and a 1-mm slit size was found to maximize the signal-tonoise ratio (SNR) while still giving reasonable signal strength. This slit size corresponded to an optical bandwidth of $1 \mathrm{~nm}$. Based on information from the NIST Atomic Spectra Database, the wavelength range formed by a bandwidth of $1 \mathrm{~nm}$ around the $541.9 \mathrm{~nm}$ (air) transition includes one other xenon ion line and two other xenon neutral lines. The $541.9 \mathrm{~nm}$ (air) transition is listed on the database as at least two orders of magnitude stronger than these three other lines so we can be reasonably assured that most of the signal (and noise) comes only from the target transition.

\section{Data Analysis and Results}

\section{A. Data Analysis}

A saturation study, shown in Fig. 5, was carried out. The plasma does not appear to be saturated by the laser.

Deconvolution of the hyperfine structure from raw LIF data was carried out using the Tikhonov regularized matrix inversion algorithm. ${ }^{18}$ This technique has the advantage of being very stable against noise so that solutions can be obtained with minimal broadening due to applied smoothing. Minimizing broadening is most important for LIF data obtained from upstream of the ionization zone where the VDFs are relatively narrow. The smoothing parameter is optimized on a per-operating-condition basis. Thus all LIF data for a given operating condition had the same degree of smoothing. The exact value of the smoothing parameter is chosen to be just high enough to eliminate noise structures with periods of $<100 \mathrm{MHz}$, or $\sim 80 \mathrm{~m} / \mathrm{s}$. However, for the operating condition of $300 \mathrm{~V}, 30 \mathrm{mg} / \mathrm{s}$, the noise level was especially high and an additional Gaussian smoothing filter of $\sim 1000 \mathrm{MHz}$ had to be applied. The hyperfine structure lineshape used in this deconvolution step was obtained in a previous study ${ }^{11}$ and shown in Fig. 6 .

The bulk velocity is calculated using particle ensemble-average. Ion acceleration is calculated by quad-fitting every three bulk velocity points and calculating the slope at the mid-point. The plasma potential is calculated from Eq. 5.

$$
\mathrm{V}_{\mathrm{p}}=\mathrm{V}_{\text {disch }}-\frac{1}{2} \frac{\mathrm{m}_{\mathrm{i}} \overline{\mathrm{v}}^{2}}{\mathrm{e}}+\mathrm{V}_{\mathrm{cg}}
$$
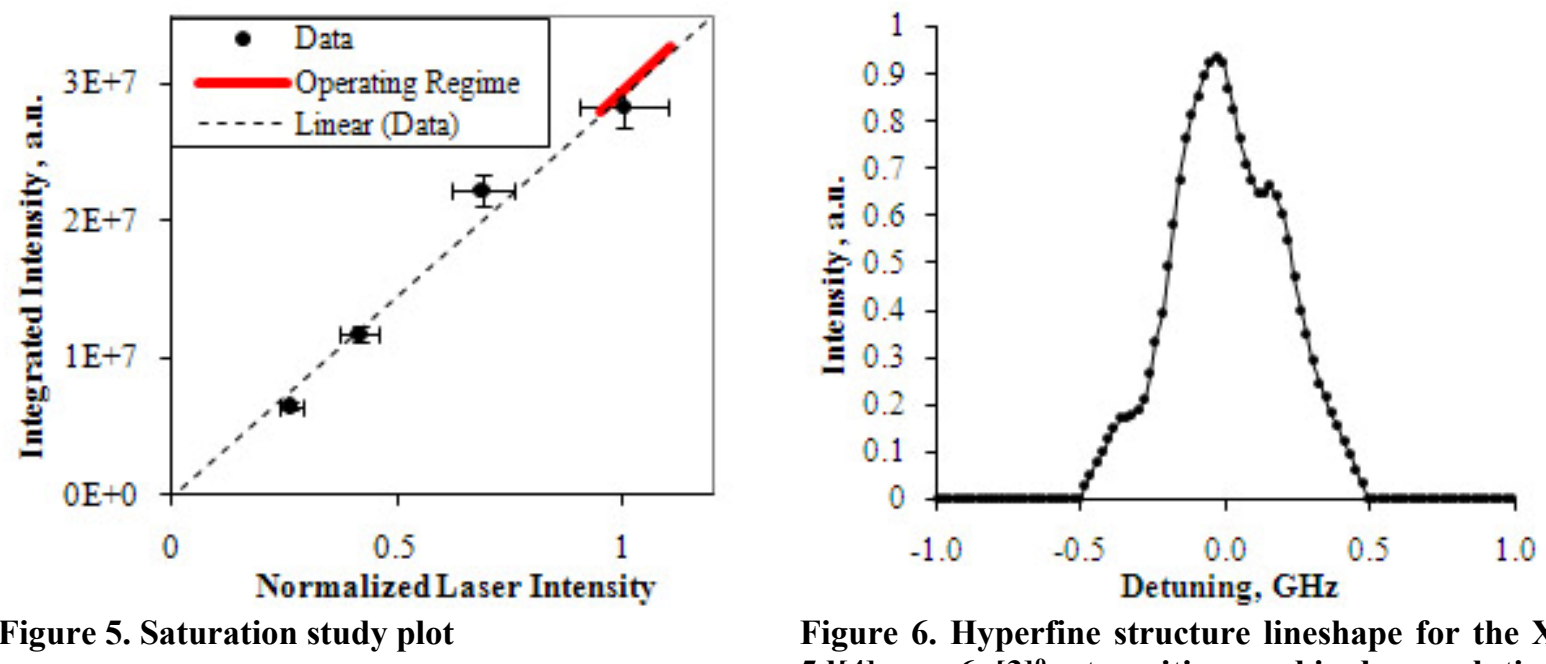

Figure 6. Hyperfine structure lineshape for the Xe II $5 \mathrm{~d}[4]_{7 / 2} \rightarrow 6 \mathrm{p}[3]^{0}{ }_{5 / 2}$ transition used in deconvolution. ${ }^{11}$ 
where $\mathrm{V}_{\text {disch }}$ is the discharge voltage, $\mathrm{m}_{\mathrm{i}}$ is the ion mass, $\overline{\mathrm{v}}$ is the bulk velocity, and $\mathrm{e}$ is the elementary charge. A correction by $\mathrm{V}_{\mathrm{cg}}$, the cathode floating voltage with respect to ground, is needed because the entire thruster floats with respect to ground. Inherent in this equation are several assumptions. First, particles are travelling only axially. This assumption is justified because measurements used for this equation are taken along the centerline of the discharge channel where the divergence of the particles is small and causes negligible cosine error. Second, the particles are singly-charged. This assumption is justified because LIF spectra are species-specific. Third, velocitychanging collisions that take place during particle acceleration can be ignored. This assumption is justifiable from a first-order analysis viewpoint because the ion mean-free-path is estimated to be on the order of $100 \mathrm{~cm}$, or 2 orders of magnitude longer than the acceleration zone. Fourth, there is negligible voltage drop between the anode and the ionization zone. This assumption is justified by plasma potential data previously taken using a fast reciprocating probe on the 6-kW thruster. ${ }^{19}$ And fifth, there is negligible amount of ionization in the acceleration zone. Although the ionization and acceleration zones are known to overlap, the overlap is small and upstream of where the bulk of acceleration takes place. With these assumptions, the electric field can also be calculated.

The axial velocity resolution is limited by the laser linewidth and is $\sim 20 \mathrm{~m} / \mathrm{s}$. The uncertainty in the calculated bulk velocity is limited by the accuracy of the stationary reference and is $\sim 50 \mathrm{~m} / \mathrm{s}$ or $2.5 \%$, whichever is greater. The uncertainty in the calculated plasma potential is $5 \%$. The uncertainty associated with the calculated acceleration and electric field is $\sim 20 \%$ due to the uncertainty associated with spatial resolution $(\sim 1.5 \mathrm{~mm})$.

\section{B. Evolution of Velocity Distribution Functions}

Figure 7 shows the evolution of the VDF for the 6-kW Hall thruster operating at $300 \mathrm{~V}, 20 \mathrm{mg} / \mathrm{s}$. This figure shows some of the basic trends observed across all tested operating conditions. The VDF starts out very thin (FWHM of $\sim 1 \mathrm{~km} / \mathrm{s}$ ) and almost Maxwellian. When the ions arrive at the acceleration zone, we begin to see distinctly non-Maxwellian VDFs with FWHM varying from 3-10 km/s. Several traces at different operating conditions also display bi-modal behavior. Figure 8 shows the more prominent bi-modal traces. The possibility that the bi-modal behavior may be linked to plasma oscillations is explored in Section V. Evolution of VDF plots for other operating conditions can be found in the Appendix.

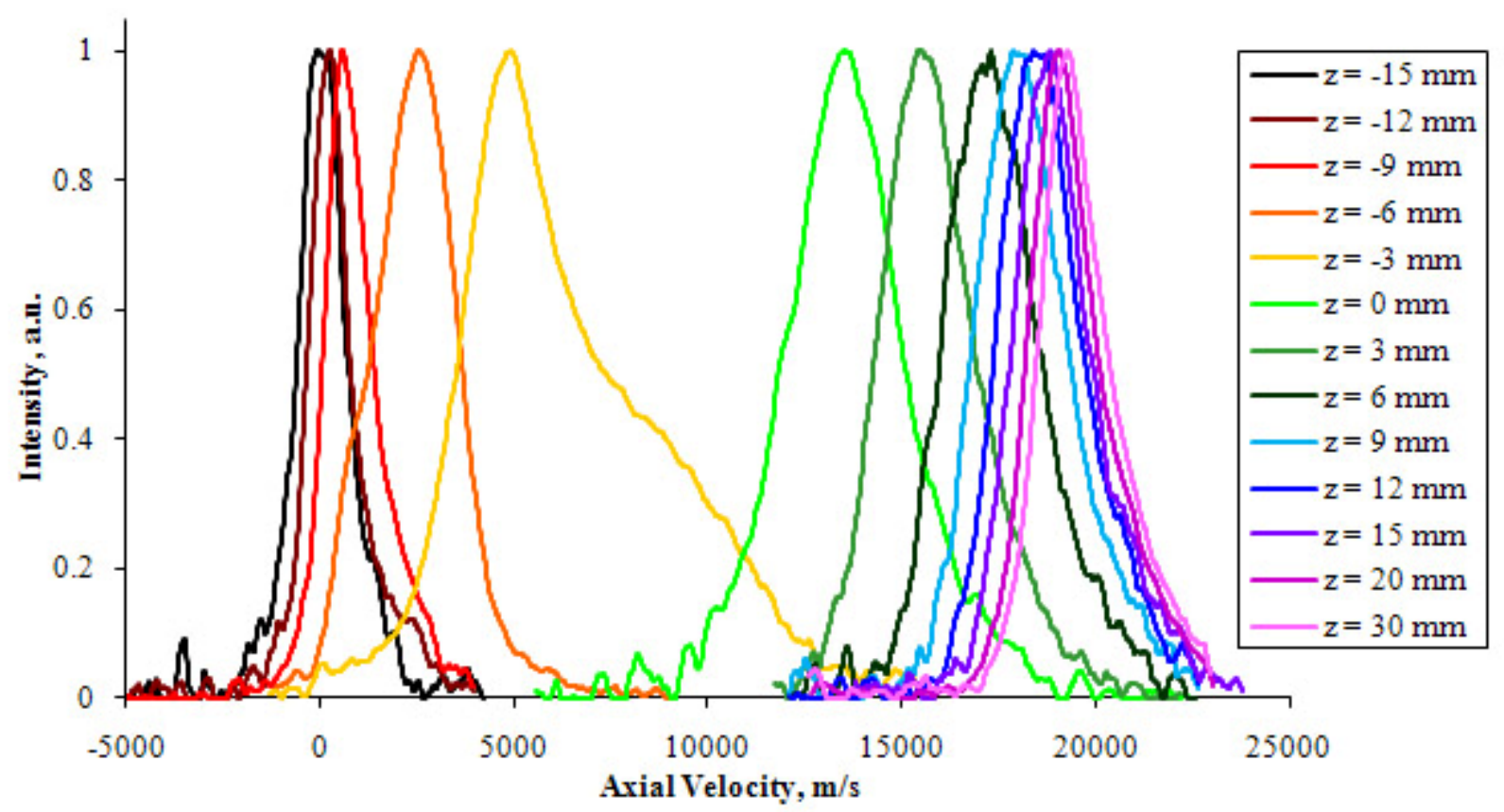

Figure 7. Evolution of velocity distribution functions for $300 \mathrm{~V}, 20 \mathrm{mg} / \mathrm{s}$ discharge. The $\mathrm{z}$ values are axial positions with negative numbers being inside the thruster and $\mathrm{z}=0 \mathrm{~mm}$ is at the exit plane. 


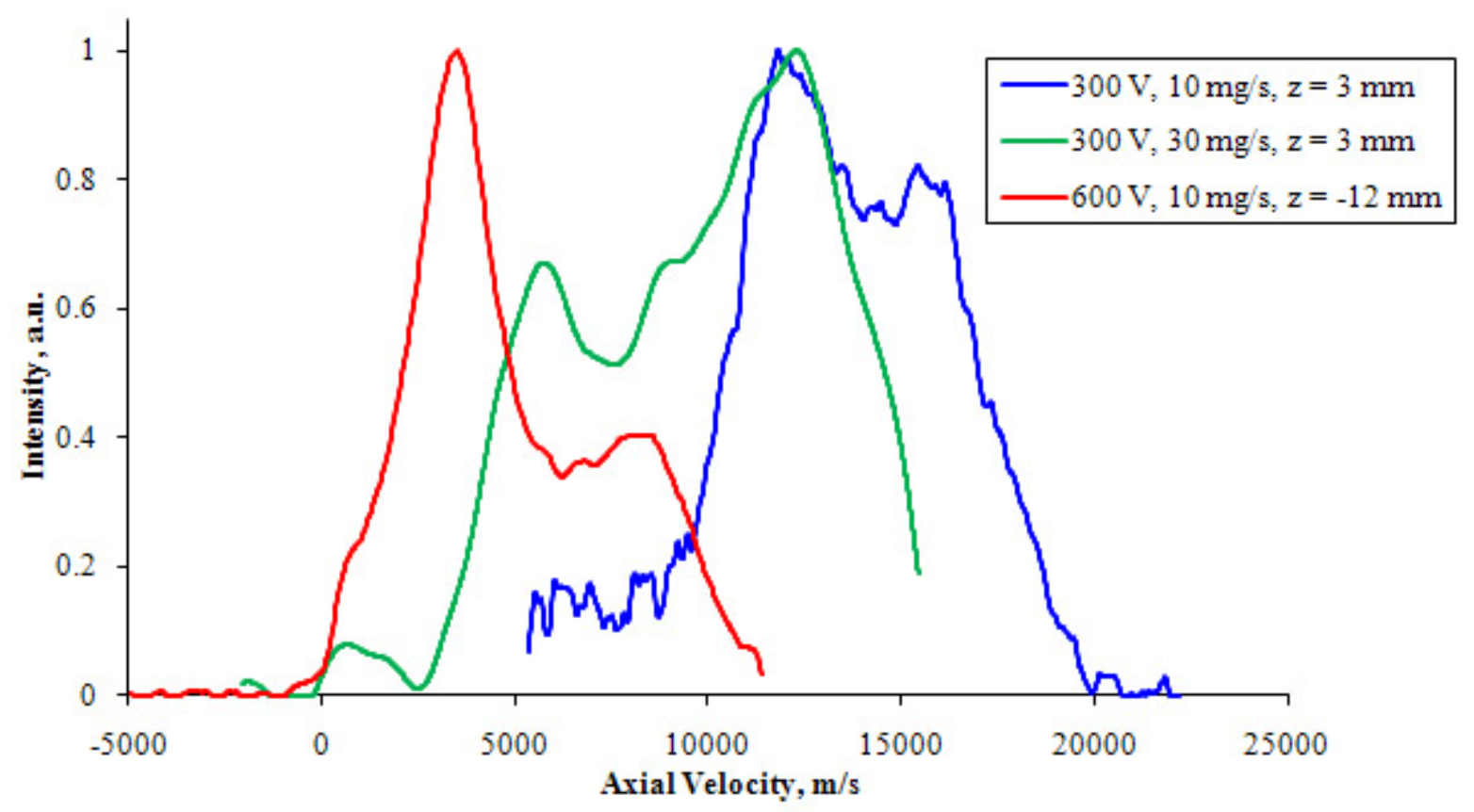

Figure 8. Several VDFs found during this experiment that showed prominent bi-modal structures.

\section{Bulk Velocity and Plasma Potential}

Figure 9 shows a plot of the bulk velocity versus the axial location for all of the tested operating conditions. The nominal operating condition displays the sharpest acceleration. While the $600 \mathrm{~V}$ condition has an extended acceleration zone compared to the $300 \mathrm{~V}$ cases, it is interesting to see that $150 \mathrm{~V}$ cases also have slightly longer acceleration zones.

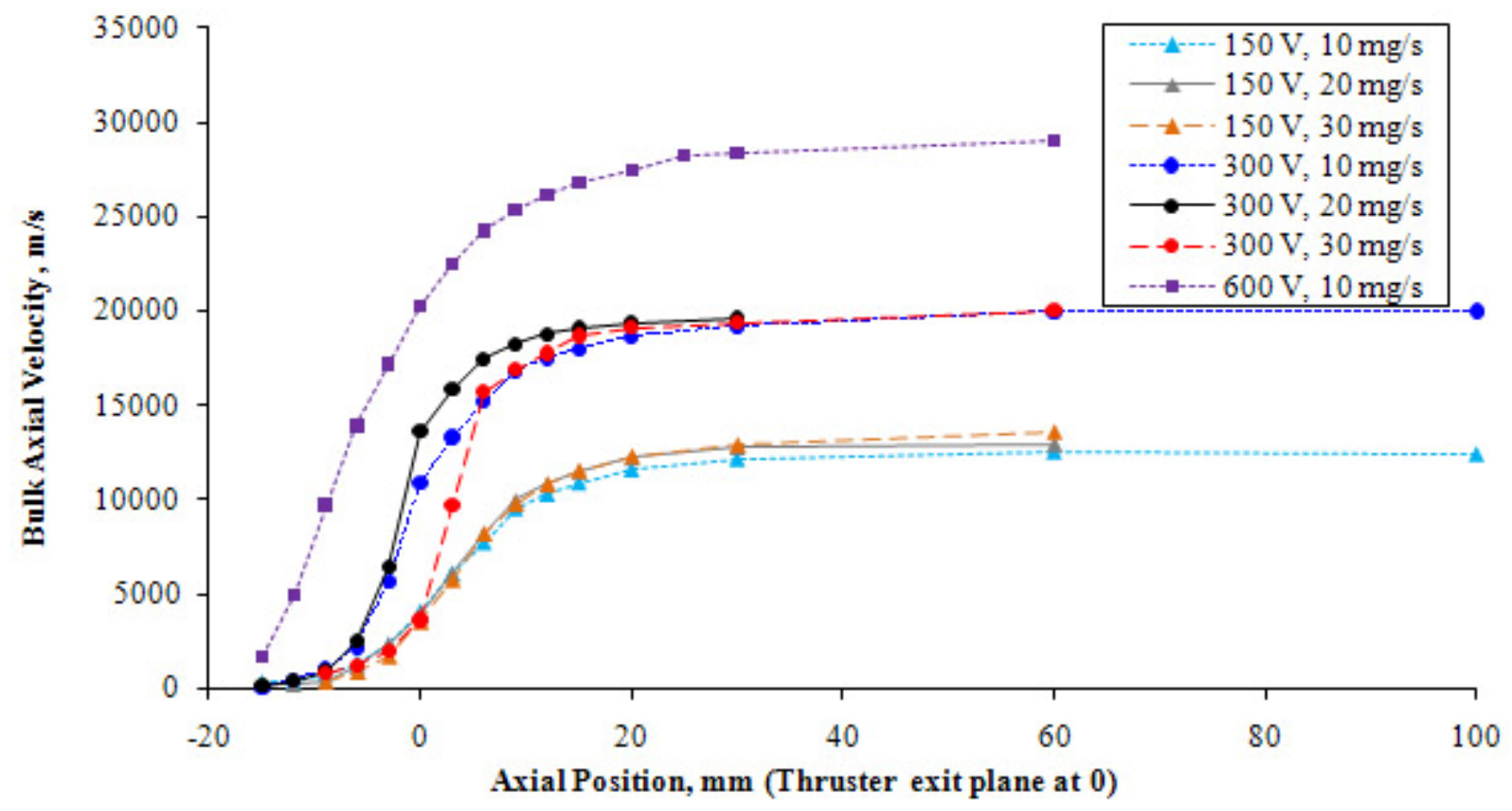

Figure 9. Bulk velocity versus position for all tested operating conditions. 
Figure 10 shows a comparison of the bulk velocity found during this experiment against a previous non-opticalfiber LIF study. ${ }^{20}$ The two sets of results match up well, thus validating the current optical-fiber-based setup.

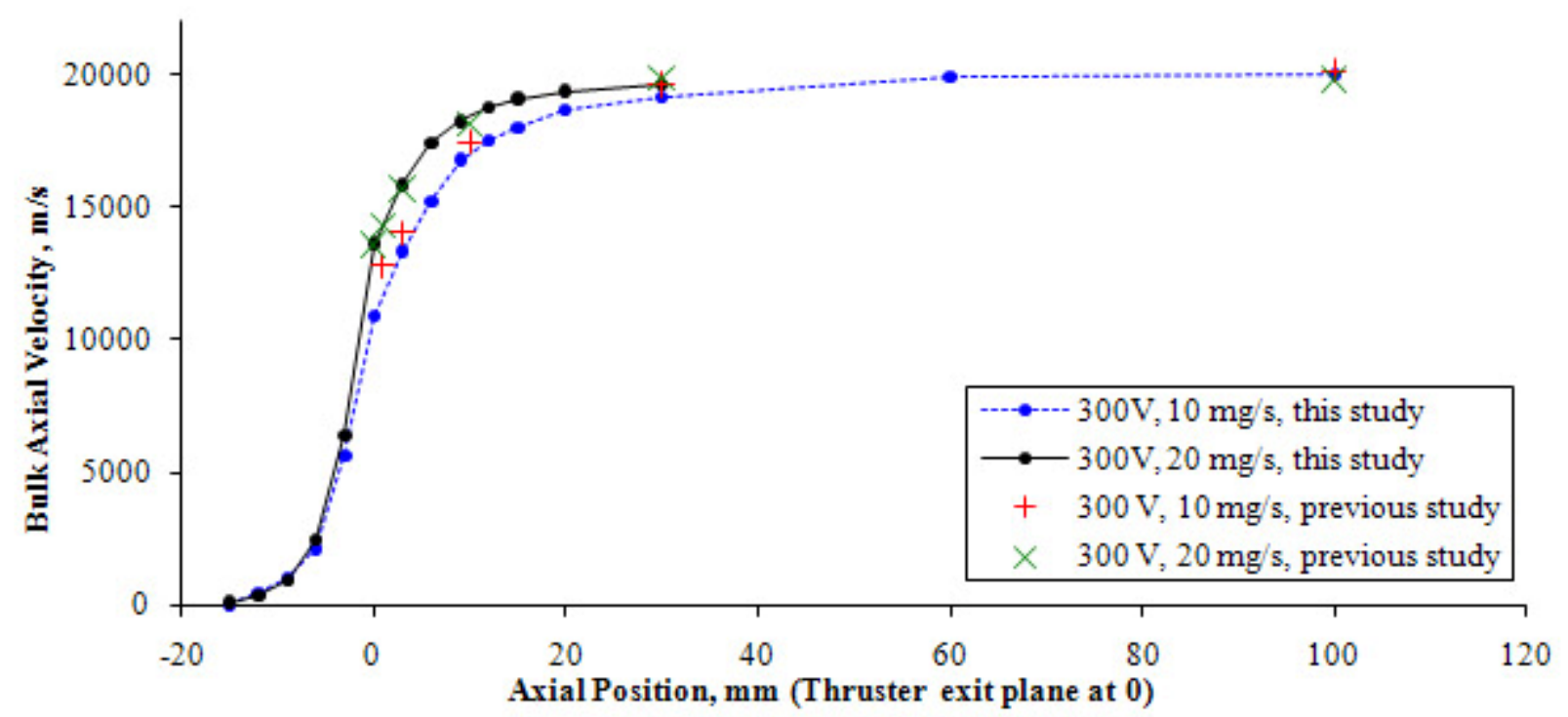

Figure 10. Comparison of LIF data obtained in this study against a previous study. ${ }^{20}$

Figure 11 shows the plasma potential calculated from the bulk velocity data. Also plotted are the plasma potential measurements from an emissive fast-reciprocating-probe study by Reid, et al. ${ }^{19}$ The best match is found for the nominal operating condition of $300 \mathrm{~V}, 20 \mathrm{mg} / \mathrm{s}$. Discrepancy in the $300 \mathrm{~V}, 10 \mathrm{mg} / \mathrm{s}$ data is current unexplained. Calculated plasma potentials for the $150 \mathrm{~V}$ and $600 \mathrm{~V}$ operating conditions can be found at the end of the appendix.

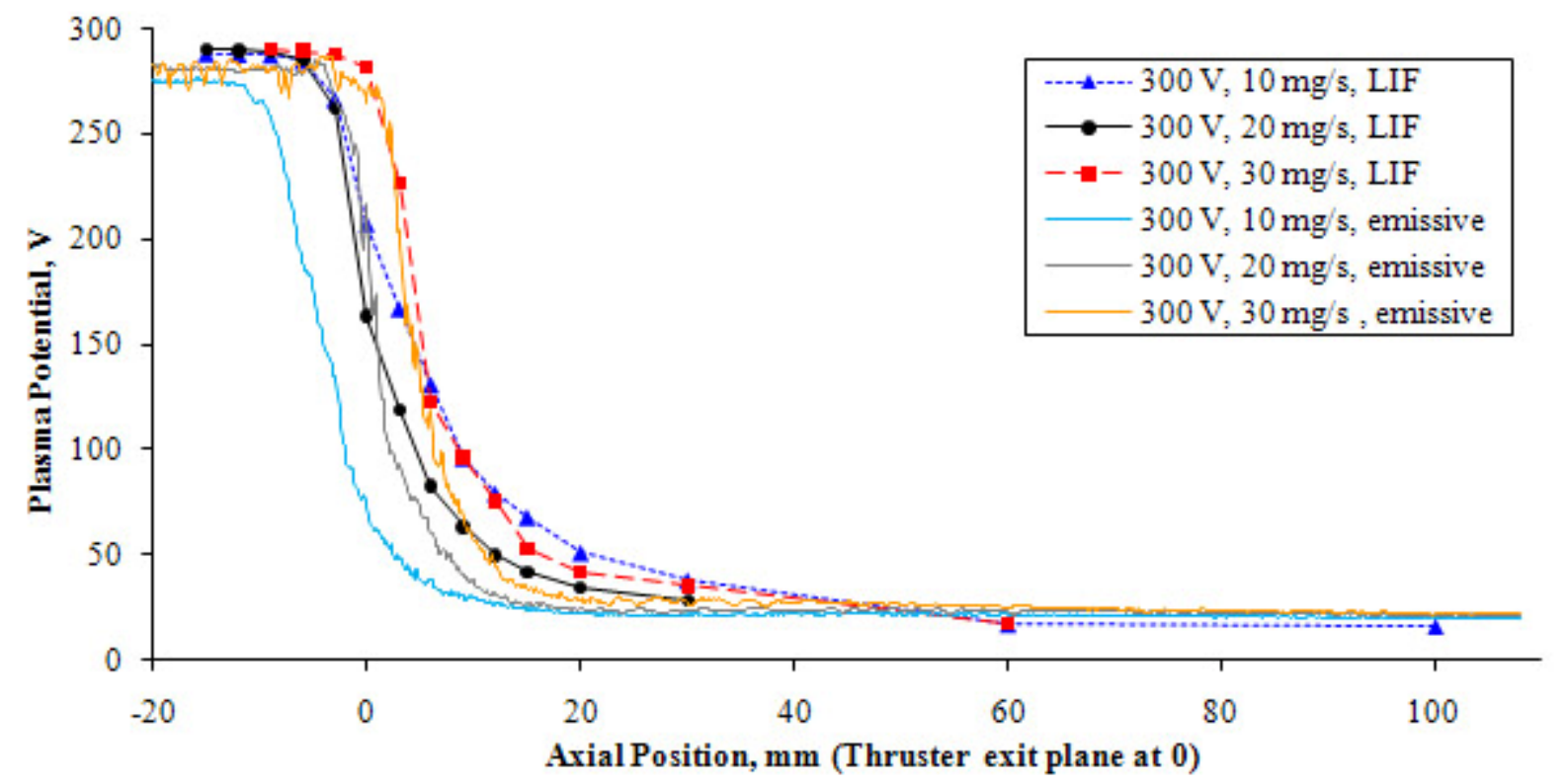

Figure 11. Calculated plasma potential for $300 \mathrm{~V}$ operating conditions, with comparison to emissive probe data. $^{19}$ 


\section{Acceleration and Electric Field}

Figure 12 shows a plot of the acceleration and the electric field as a function of position for all tested conditions. Given the assumptions listed for Eqn. 5, the acceleration and the electric field have a proportional relationship and are thus plotted together. Table 2 lists the acceleration zone length, which is defined as the distance it takes for the bulk velocity to go from $10 \%$ to $90 \%$ of the maximum bulk velocity. Also listed is the acceleration zone location, defined as the average of the $10 \%$ - and $90 \%$-of-maximum bulk velocity locations. Lastly, the position where peak acceleration occurs is listed. In general, the acceleration zone is more recessed into the channel at higher discharge voltages and lower anode mass flow rate though the relationship with mass flow rate is not as clear. The peak acceleration position tends to be further into the channel than the location of the acceleration zone, suggesting that more of the potential drop occurs nearer upstream than downstream.

With the information from Table 2, we can now re-process Fig. 9 to look for universal features in the acceleration zone. Figure 13 is a normalized plot of bulk velocity versus position. The bulk velocity is normalized against the maximum recorded bulk velocity for each condition. The axial position is normalized against the acceleration zone length as previously defined. The zero of the normalized axial position is set at the position where the bulk velocity reaches $10 \%$ of the maximum bulk velocity, which is also the start of the acceleration zone as previously defined. Note that the seven curves overlap each other very well suggesting a near-identical accelerationzone structure for all tested operating conditions. The slopes of the curves are steeper near the start of the acceleration zone than near the end. This means more particle acceleration takes place in the first half of the acceleration zone than in the second half.

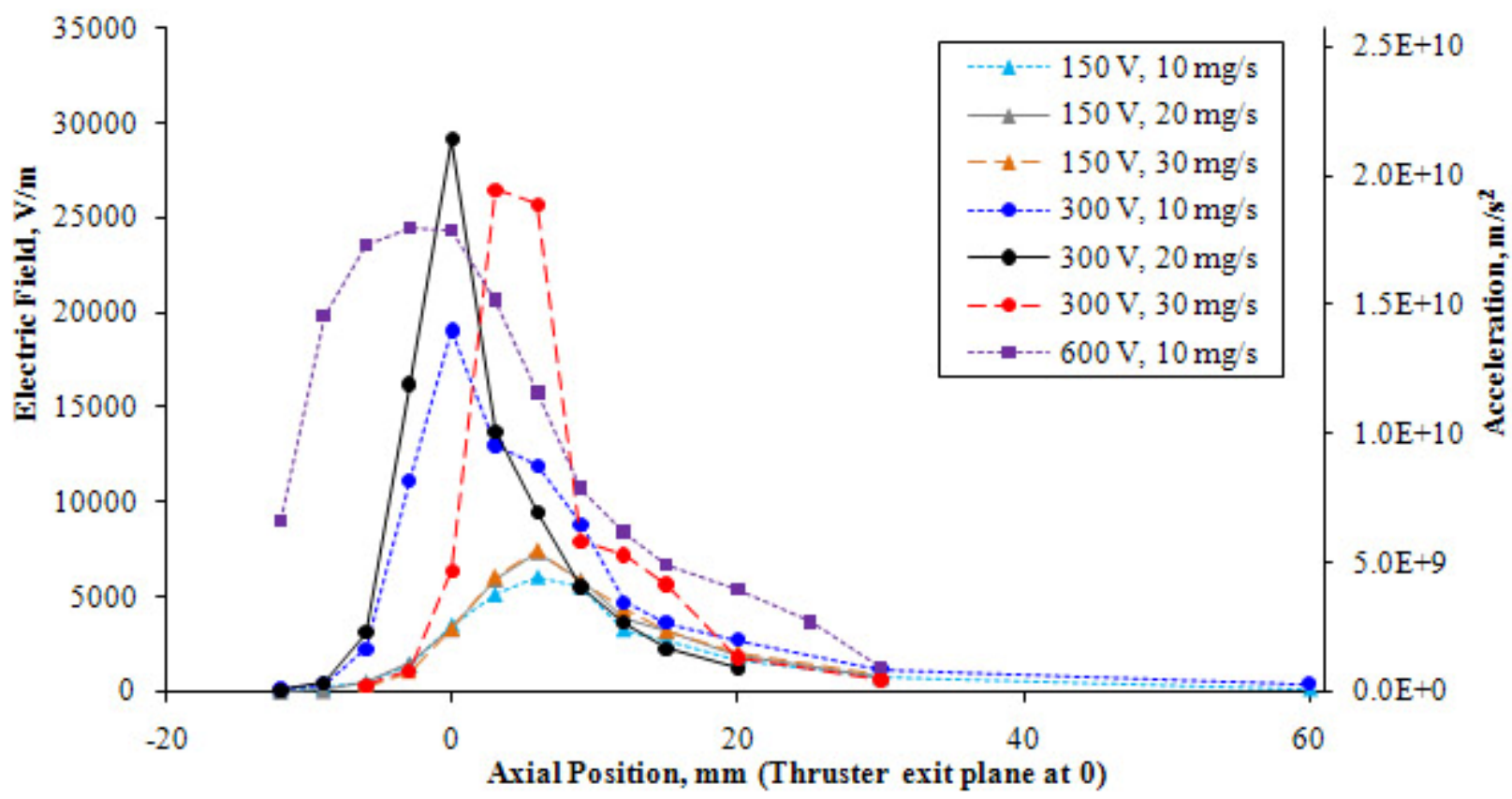

Figure 12. Acceleration and electric field calculated from bulk velocity for all tested conditions.

11

American Institute of Aeronautics and Astronautics 


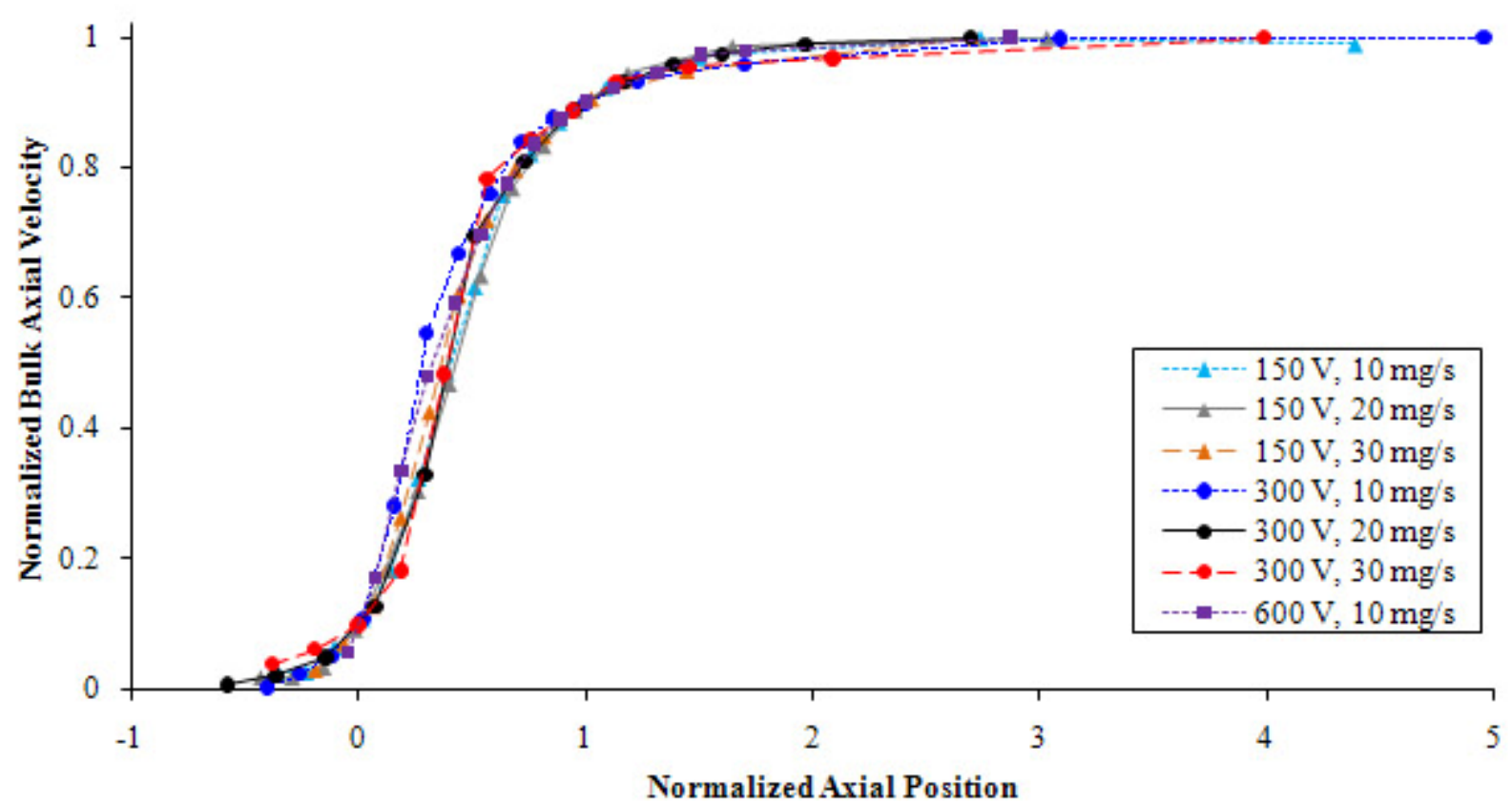

Figure 13. Normalized bulk axial velocity versus normalized axial position. Bulk velocity is normalized against maximum bulk velocity reached for each condition. Axial position is normalized against acceleration zone length with 0 being the start of the acceleration zone.

\section{Noise Analysis}

Normally, noise is the undesirable part of LIF data that should be removed where possible. However, for studying plasma oscillations, noise can give a qualitative description of the magnitude of the oscillations. While plasma oscillations typically have characteristic frequencies and fluctuation patterns, the combination of the many types of plasma oscillations in a Hall thruster will make the overall fluctuation pattern seem random. To use an analogy, if each type of plasma oscillation is represented by a single musical melody, playing all of these plasma melodies at once will sound like a cacophony, or in other word, noise. As such, the magnitude of the noise in LIF data scales with the overall magnitude of the plasma oscillations. However, LIF noise amplitude is difficult to characterize and it is easier to study signal-to-noise ratio (SNR). Equation 6 shows the definition of SNR used in this paper. To make this definition independent of intensity scaling, the raw data are normalized against its peak intensity value before the SNR is calculated. Thus, the numerator is always 1.

$$
\mathrm{SNR} \equiv \frac{\text { Amplitude of tallest peak }}{\text { Root mean square of noise }}=\frac{1}{\text { Noise }_{\text {RMS }}}
$$

Note that there are other sources of noise present in LIF data. To make sure noise due to plasma oscillations is dominant over all other sources, we took LIF traces with the thruster off and compared them to when the thruster is on. We note that the traces with the thruster on show noise levels that are typically 1 to 3 orders of magnitude higher than thruster-off traces depending on the severity of the plasma oscillations.

Using SNR instead of characterizing noise has the disadvantage that the SNR depends on the signal amplitude. If the noise level remains unchanged throughout the entire acceleration zone, the SNR will peak where the ion density is the highest and drop off at far upstream and far downstream positions. This trend arises because far upstream of the acceleration zone, not many ions have been formed yet, and far downstream the ions are fanning out at high speed, decreasing the ion density.

Figure 14 shows a plot of the SNR as a function of position for all tested operating conditions. Most of the curves display drop-offs toward far upstream or far downstream positions as expected. The $600 \mathrm{~V}, 10 \mathrm{mg} / \mathrm{s}$ condition did not show this drop-off far upstream because we were not probing far enough upstream. Unexpectedly, 
the SNR drops by quite a bit in the middle of the acceleration zone at seemingly random locations. We believe these locations are areas of high plasma oscillations because sudden drops in SNR in these zones can only be attributed to sudden rise in noise level. Since plasma oscillations, with some exceptions, affect the movement of electrons much more than ions, the oscillations in these electrons will translate to fluctuations in the photons released by electronimpact excitation, which is picked up as noise by the experimental setup. Furthermore, these locations coincide with the regions in which the Hall current is known, from a previous study ${ }^{21}$, to reside. The electric field is strong in these regions; large amounts of energy are being dumped into the charged species. Some of the curves (e.g., $300 \mathrm{~V}, 20$ $\mathrm{mg} / \mathrm{s}$ and $600 \mathrm{~V}, 10 \mathrm{mg} / \mathrm{s}$ ) display two zones where the SNR drops. The reason for this is not clear. We hypothesize that each of these regions may represent a different set of plasma oscillations at work.

From Fig. 14, we also see that the SNR goes down as the anode mass flow rate goes up while discharge voltage has no obvious effect. This trend holds despite the fact that higher mass flow rates correspond to higher ion densities. The SNR for the LIF traces taken at $30 \mathrm{mg} / \mathrm{s}$ conditions are over an order of magnitude lower than SNR for the $10 \mathrm{mg} / \mathrm{s}$ traces.

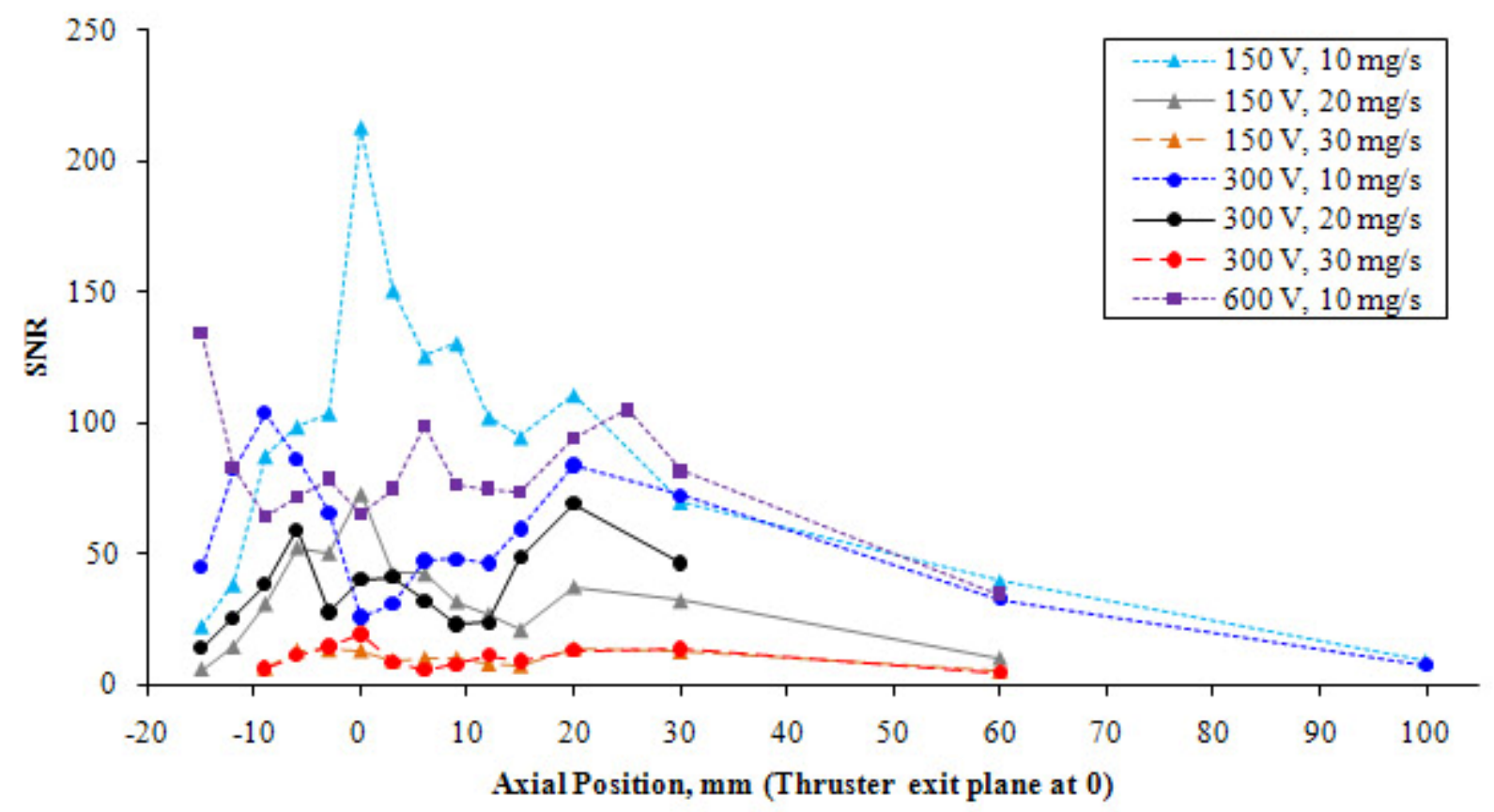

Figure 14. Signal-to-noise ratio versus axial position for all tested operating conditions.

\section{Instantaneous Bulk Velocity PDF Extraction}

If we make the assumption that the instantaneous stationary VDF is not dependent on time and the plasma is quasi-steady, then Eqn. 4 can be rewritten as Eqn. 7, and the extraction of the instantaneous bulk velocity PDF is a simple deconvolution operation.

$$
\bar{g}(v)=\int_{-\infty}^{\infty} f(v-\hat{v}) \operatorname{pdf}(\hat{v}) d \hat{v}=f(v) \otimes \operatorname{pdf}(v)
$$

We further assume that the VDF obtained far upstream of the acceleration zone is representative of $f(v)$ and that this VDF is approximately Maxwellian. We then deconvolve the said VDF from downstream VDFs via Tikhonov regularization ${ }^{18}$ to obtain the PDFs. Figure 15 shows the furthest upstream VDF obtained for the $300 \mathrm{~V}, 20 \mathrm{mg} / \mathrm{s}$ condition compared to the Maxwell-Boltzmann equation fitted to the VDF. The fit serves as the basis for deconvolution. Figure 16 shows the resulting PDFs from the deconvolution operation compared with the original VDFs for two difference axial positions. Both VDFs are from the $300 \mathrm{~V}, 20 \mathrm{mg} / \mathrm{s}$ condition with the wider one from an axial position of $-3 \mathrm{~mm}$ and the thinner one from an axial position of $15 \mathrm{~mm}$. These two VDFs were chosen because they are representative of the widest and narrowest VDFs found during this experiment. 


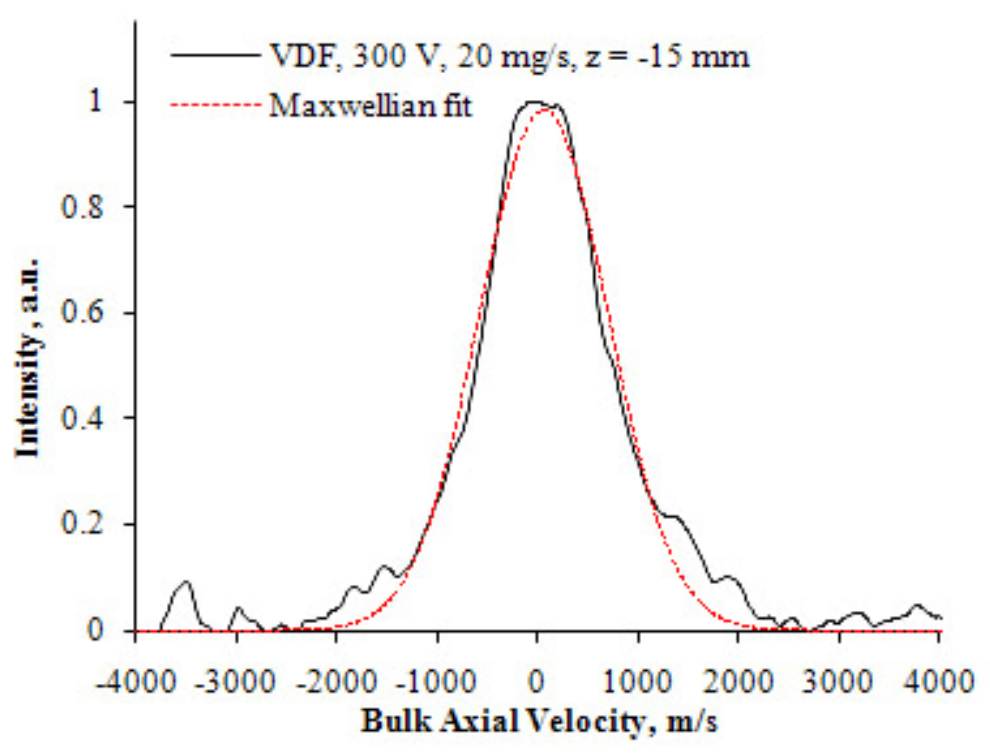

Figure 15. Comparison between pre-accelerated VDF and
The smoothing parameter had to be chosen on a scan-by-scan basis because of the variability in the noise level. Too high of a smoothing parameter meant the PDF becomes un-realistically wide. While too low of a smoothing parameter meant ringing effects start to show up, distorting the PDF lineshape. This ringing effect becomes more pronounced for scans whose width and shape is similar to the original Maxwellian fit used as the deconvolution basis. For this reason, far upstream and far downstream scans were not processed. The smoothing parameter was chosen to minimize ringing, though a little bit of ringing can still be seen in Fig. 16 for the $\mathrm{z}=15 \mathrm{~mm}$ PDF where the curve overshoots and hits the $\mathrm{x}$-axis on the left wing.

\section{Maxwellian fit.}

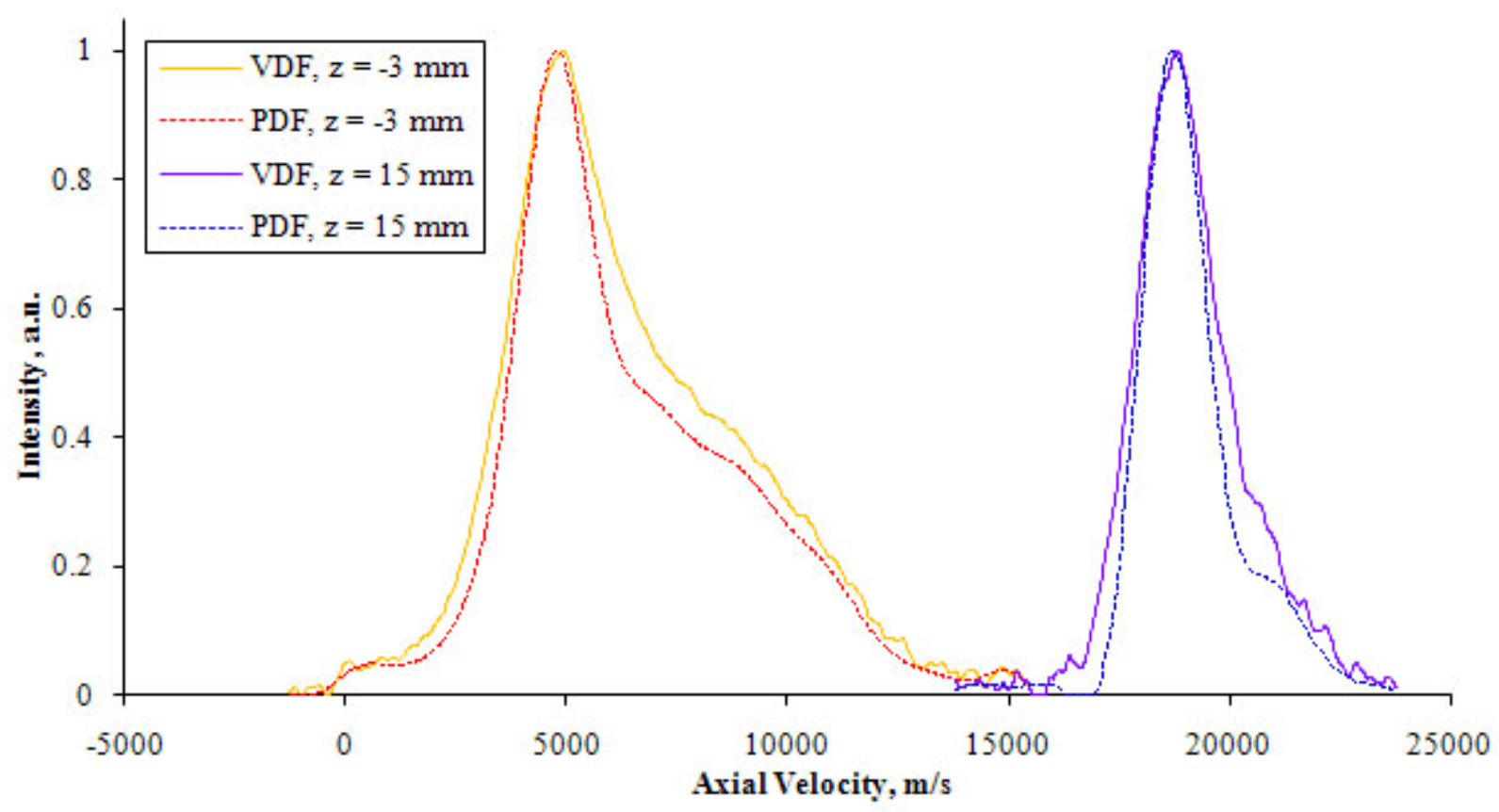

Figure 16. Comparison of two sets of VDFs and PDFs for the operating condition of $300 \mathrm{~V}, 20 \mathrm{mg} / \mathrm{s}$. 
Figure 17 shows a contour plot of the instantaneous bulk velocity PDF obtained for the $300 \mathrm{~V}, 20 \mathrm{mg} / \mathrm{s}$ condition. Unfortunately, the number LIF traces in axial space is not dense enough to generate a proper contour, which is why there are several red spots on the left side of the plot that should have connected up into a band.

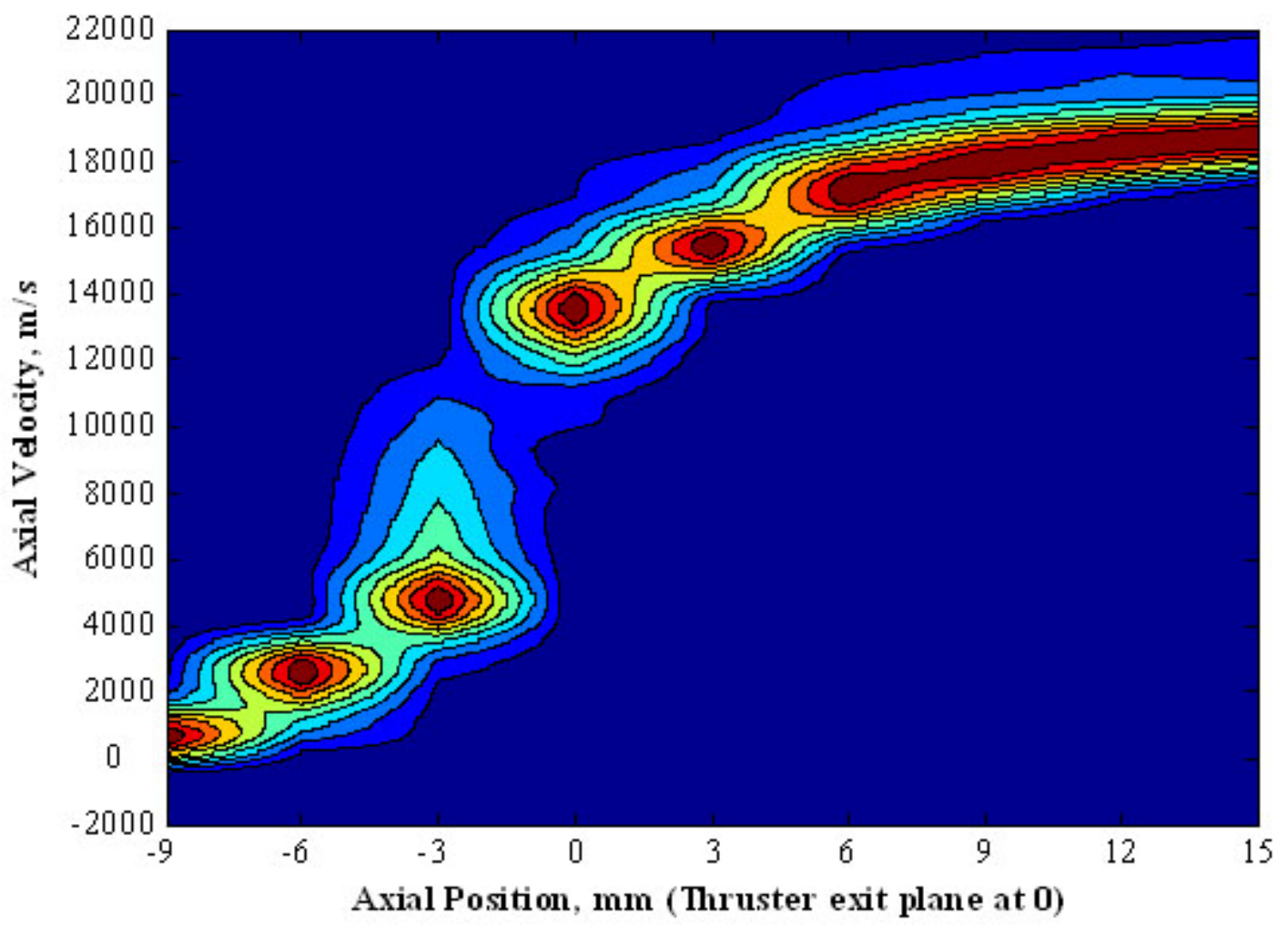

Figure 17. Contour map of instantaneous bulk velocity PDF obtained for the $300 \mathrm{~V}, 20 \mathrm{mg} / \mathrm{s}$ condition, red corresponds to high probability while blue corresponds to low probability.

Figure 18 is plotted to better show, qualitatively, how the instantaneous bulk velocity varies in velocity and physical space. In Fig. 18, the pairs of lines represent the upper and lower value of the FWHM velocities for each scan locations. In other words, if one draws a half-max horizontal line through a PDF, the two intersections represent a qualitative mean of determining the width and location of the PDF. One of the lines for each operating condition connects up the higher velocities, while the other line connects up the lower velocities. The in-between region is where one is most likely to find the instantaneous bulk velocity in velocity and physical space. Note the lines only branch into pairs after the $-9 \mathrm{~mm}$ axial position because the VDFs were not significantly broadened upstream of this position and the bulk velocity is plotted instead.

There are several possible explanations for the shape we see in Fig. 18. But based on existing physical knowledge about the Hall thruster, we believe the most likely explanation is that we are seeing the acceleration zone move back and forth in space as a result of the breathing mode oscillation. From basic plasma physics, we know that for a quasi-neutral plasma the plasma potential profile is largely fixed by the conductivity profile. In the Hall thruster, the starting point for this potential profile is fixed by the ionization zone. We know that the basic premise of the breathing mode involves an ionization zone that oscillates in the axial direction. It is not unreasonable to conjecture that the acceleration zone follows this oscillation in space. This explanation also fits well with the observed bi-modal behavior if we assume the breathing mode oscillation is close to sinusoidal in nature. An object that undergoes sinusoidal oscillation spends more time at the two extremes of its range of motion. This behavior gives rise to two peaks when averaged over time. If the above explanation holds, we see that the acceleration zone only moves back and forth over a spatial extend of about $1-2 \mathrm{~mm}$ in the axial direction for the nominal operating condition, but moves over a spatial extend of $2-4 \mathrm{~mm}$ for the $300 \mathrm{~V}, 10 \mathrm{mg} / \mathrm{s}$ and $30 \mathrm{mg} / \mathrm{s}$ conditions.

Another possible explanation is that the ions were heated up by the bombarding electrons. This is likely a contributing factor to the broadening of the VDFs, but it is not likely to be the primary factor. If the broadening was 


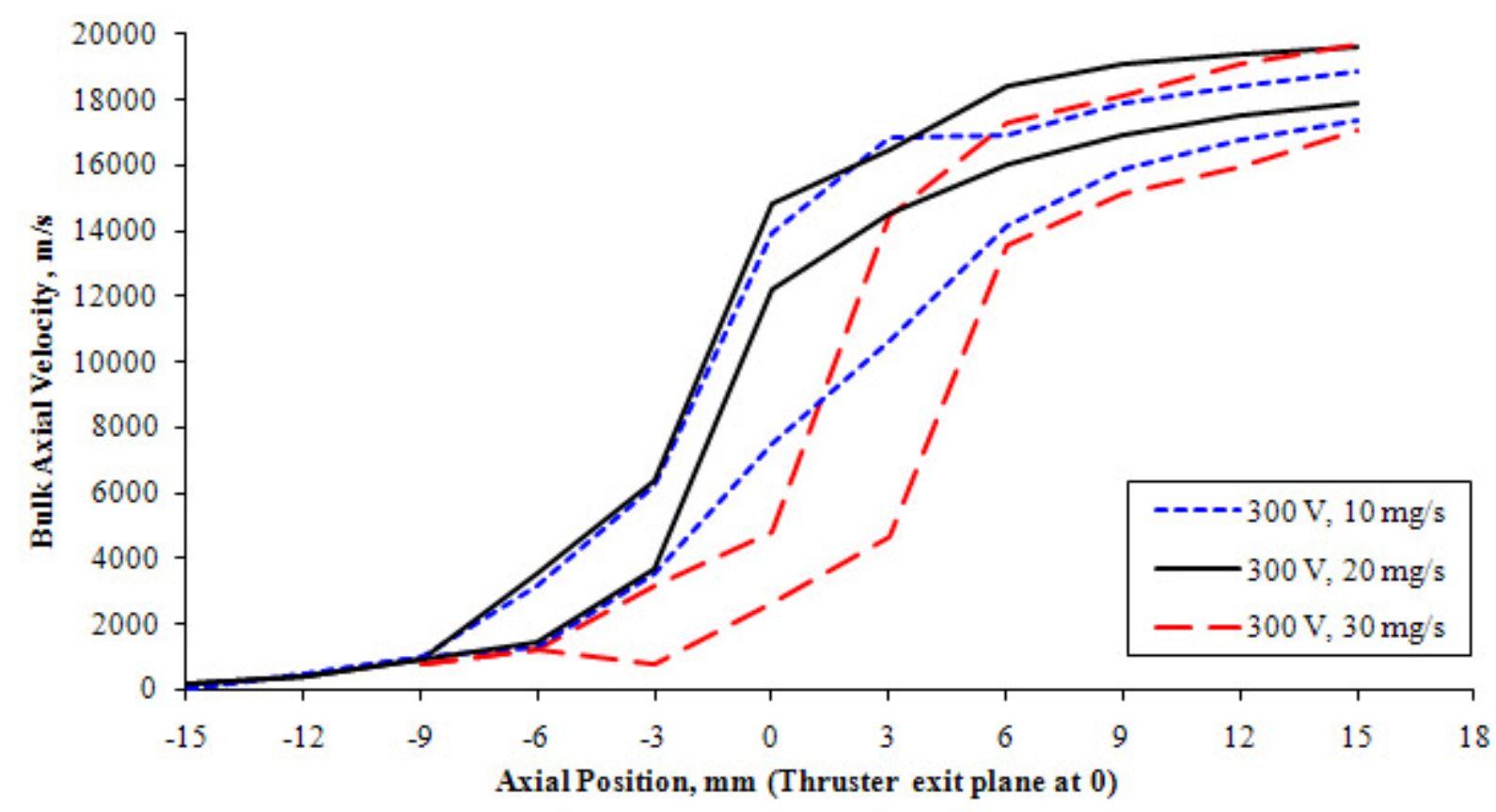

Figure 18. FWHM boundaries of the instantaneous bulk velocity PDFs

due entirely to heating, the VDFs downstream of Hall current should be much broader than what is measured even with kinematic compression taken into account. For example, for the $300 \mathrm{~V}, 30 \mathrm{mg} / \mathrm{s}$ condition, the particles at $\mathrm{z}=3$ $\mathrm{mm}$ has a bulk velocity of $9700 \mathrm{~m} / \mathrm{s}$ and a FWHM velocity of $9900 \mathrm{~m} / \mathrm{s}$. If the broadening of this population is purely a result of electron bombardment heating, accounting for kinematic compression, the projected FWHM velocity at $\mathrm{z}=60 \mathrm{~mm}$ should have been $4700 \mathrm{~m} / \mathrm{s}$. Yet, the LIF measurement at $\mathrm{z}=60 \mathrm{~mm}$ shows a FWHM velocity of only $2800 \mathrm{~m} / \mathrm{s}$.

It is also possible that we cannot assume the instantaneous stationary VDF is independent of time. This component of the time-averaged VDF may undergo a complex evolution as it moves back and forth in physical space. New slow ions are also being added to the ion population in small amounts due to the overlap in the ionization and acceleration zones. Current LIF measurement capabilities cannot prove or disprove this assumption. However, we put forth the argument that any variability in the instantaneous stationary VDF is relatively small because such variability in velocity can only come about as a result of collisions. The ion mean-free-path is on the order of $10 \mathrm{~cm}$ to $1 \mathrm{~m}$, much longer than the length of the acceleration zone. And relatively few ionizing collisions take place where the bulk of the acceleration takes place.

Lastly, it is possible that the broadening in VDFs is due to some other plasma oscillations. This is difficult prove without some form advanced transient diagnostics. However, we put forth the argument that the breathing mode is at least an order of magnitude more energetic than other plasma oscillations based on discharge current power spectra shown in Fig. 19 in the next section.

Note that the instantaneous bulked velocity PDFs for the $150 \mathrm{~V}$ and $600 \mathrm{~V}$ operating conditions were not extracted because we believe we should make further improvement to our deconvolution techniques before extracting them, and that the PDFs for the $300 \mathrm{~V}$ operating conditions were sufficient for the purposes of this paper.

We also did not evolve the upstream VDF by kinematic compression before deconvolving them from the downstream VDF because any error introduced will be small. Since we do not know how kinematic compression competes with heating effects, it is thought best to leave the analysis simple and the results qualitative. 


\section{Discharge Current Power Spectra}

Complimentary discharge current power spectra were also taken during a separate study, in which all operating conditions and thruster parameters were matched to those used in this study. In particular, the discharge current was matched to within $1 \%$, limited by uncertainty associated with the mass flow controller. The data was collected using a Bell NT-50 shunt and a Tektronix TDS-3034B oscilloscope. Each power spectrum is based on an ensemble average of eight fast Fourier transformed traces. Figure 19 shows the discharge current power spectra. For most of the tested conditions, the breathing mode shows up as a clear spike between 5 to $25 \mathrm{kHz}$. For the nominal condition, this spike is smoothed out suggesting a more subdued breathing mode. The $300 \mathrm{~V}, 30 \mathrm{mg} / \mathrm{s}$ shows a different pattern from the other traces. Not only is the breathing mode hump spread out over a large frequency space (assuming it is a breathing mode hump), there is also a second hump at $\sim 70 \mathrm{kHz}$. Combined with the fact that $300 \mathrm{~V}, 30 \mathrm{mg} / \mathrm{s}$ is much noisier than the other operating conditions, there are likely very complex plasma mechanics going on.

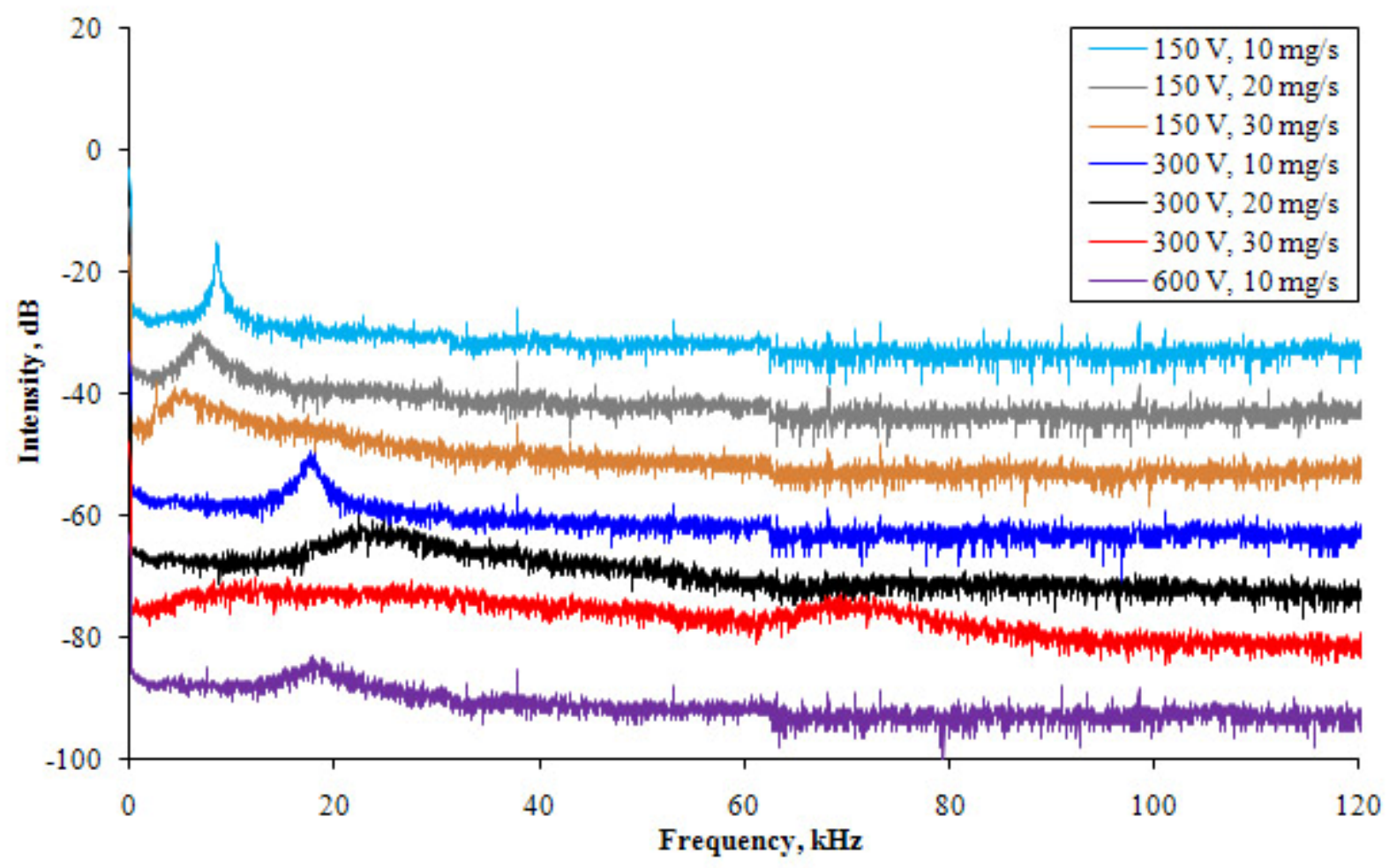

Figure 19. Discharge current power spectra. Intensity scaling is based on a 10-dB logarithmic progression. The second trace listed in the legend has a $-10 \mathrm{~dB}$ offset with respect to the first trace listed. Each successive trace has an additional $-10 \mathrm{~dB}$ offset from the preceding trace in the legend list.

\section{Conclusion and Future Works}

We have performed a singly-charged xenon LIF experiment probing inside and in the near-field of the 6-kW Hall thruster discharge channel. The new optical fiber setup used in this experiment was validated against a previous setup. Lengths of the acceleration zones for the seven tested operating conditions were found to vary from 14 to 26 $\mathrm{mm}$. The nominal operating condition of $300 \mathrm{~V}$ discharge voltage, $20 \mathrm{mg} / \mathrm{s}$ anode mass flow showed the shortest acceleration zone while the $600 \mathrm{~V}, 10 \mathrm{mg} / \mathrm{s}$ case showed the longest. Location of the acceleration zone tends to move into the channel as the discharge voltage increased and out of the channel as the anode mass flow rate increased. More of the acceleration takes place in the upstream half of the acceleration zone than in the downstream half. The normalized plot of the acceleration zone showed that all of the operating conditions have a very similar acceleration structure.

Effects of plasma oscillations on time-averaged LIF results were analyzed using three different approaches. First, the plot of the signal-to-noise ratio shows that noise caused by plasma oscillations tends to be much stronger in the middle of the acceleration zone than on the edges. The signal-to-noise ratio is also much lower for the higher anode 
mass flow rate conditions when compared to that of the lower anode mass flow rate conditions. Second, an analysis of the instantaneous bulk velocity PDF supports the idea that the acceleration zone oscillates in space. The cause is attributed to the breathing mode though other causes are also discussed. The acceleration zone oscillates over a range of $\sim 1-2 \mathrm{~mm}$ for the nominal operating condition, and $\sim 2-4 \mathrm{~mm}$ for the $300 \mathrm{~V}, 10 \mathrm{mg} / \mathrm{s}$ and $30 \mathrm{mg} / \mathrm{s}$ conditions. Third, discharge current power spectra shows that the breathing mode dominates over other forms of plasma oscillations for most of the operating conditions. The frequency of the breathing mode varies from $5-25 \mathrm{kHz}$.

To properly construct the instantaneous bulk velocity PDF, we need to improve the spatial resolution and take denser LIF scans where the velocity undergoes the most rapid changes. A better deconvolution routine that produces less ringing effect in the resulting PDF would be highly desirable. Improving the signal-to-noise ratio will decrease the amount of smoothing needed and improve the fidelity of the PDF results. Properly constructing the instantaneous bulk velocity PDF is a worthwhile effort because we can ultimately convert it into a plasma potential PDF in order to learn more about the physics of the Hall thruster plasma. 


\section{Appendix}

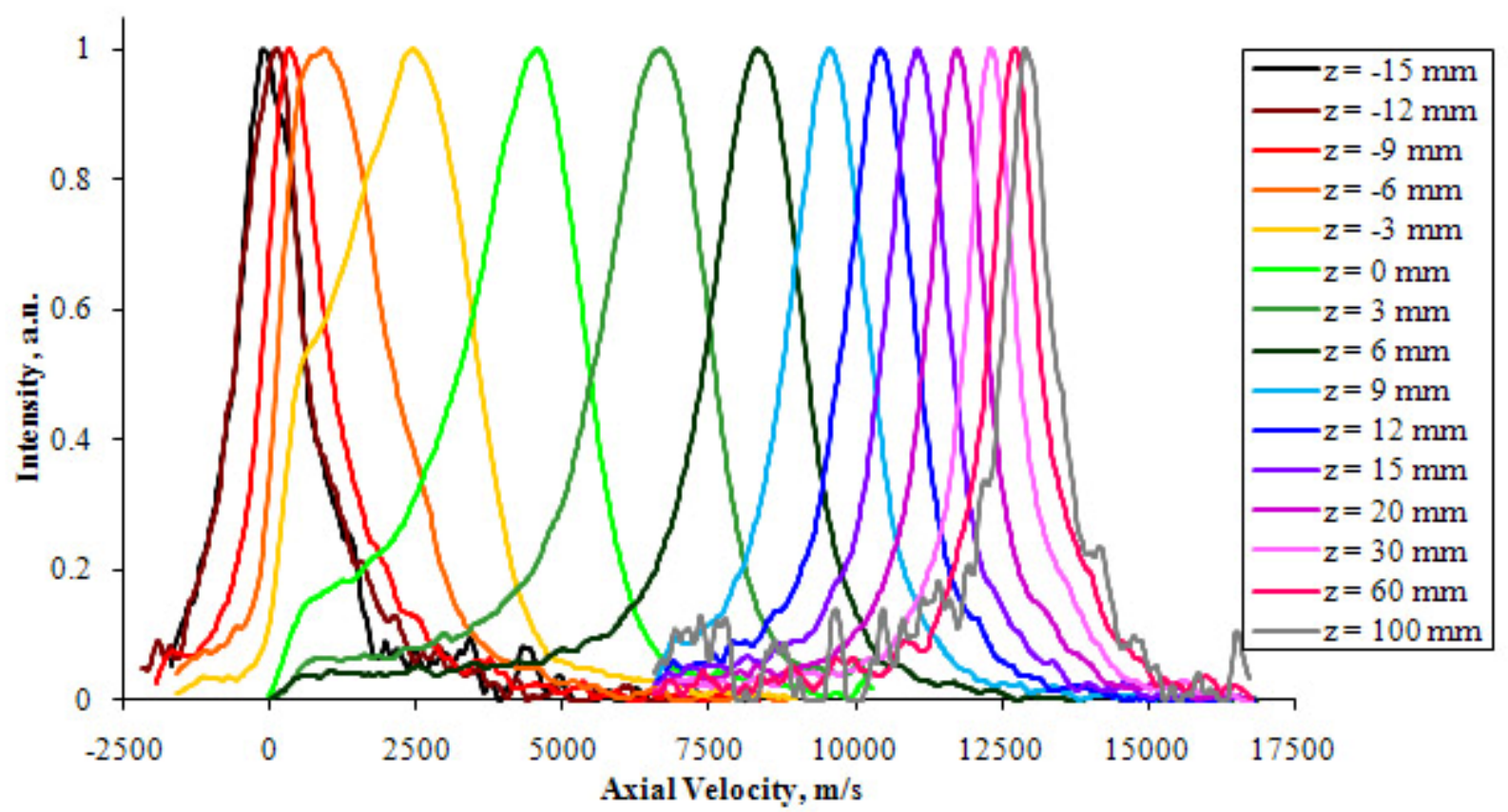

Figure 20. Evolution of velocity distribution functions for $150 \mathrm{~V}, 10 \mathrm{mg} / \mathrm{s}$ discharge. The $\mathrm{z}$ values are axial positions with negative numbers being inside the thruster and $\mathrm{z}=0 \mathrm{~mm}$ is at the exit plane.

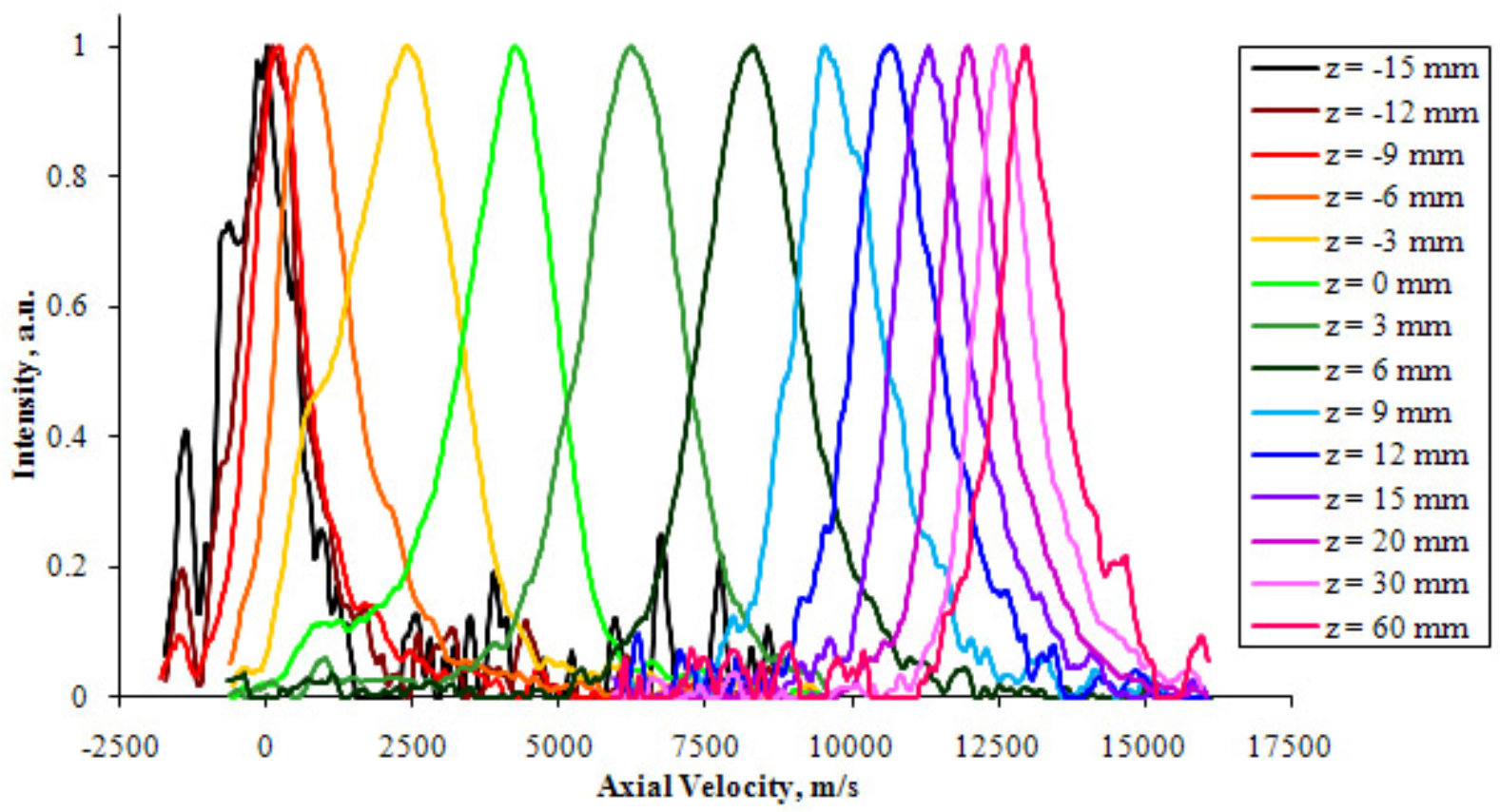

Figure 21. Evolution of velocity distribution functions for $150 \mathrm{~V}, 20 \mathrm{mg} / \mathrm{s} \mathrm{discharge.}$ 


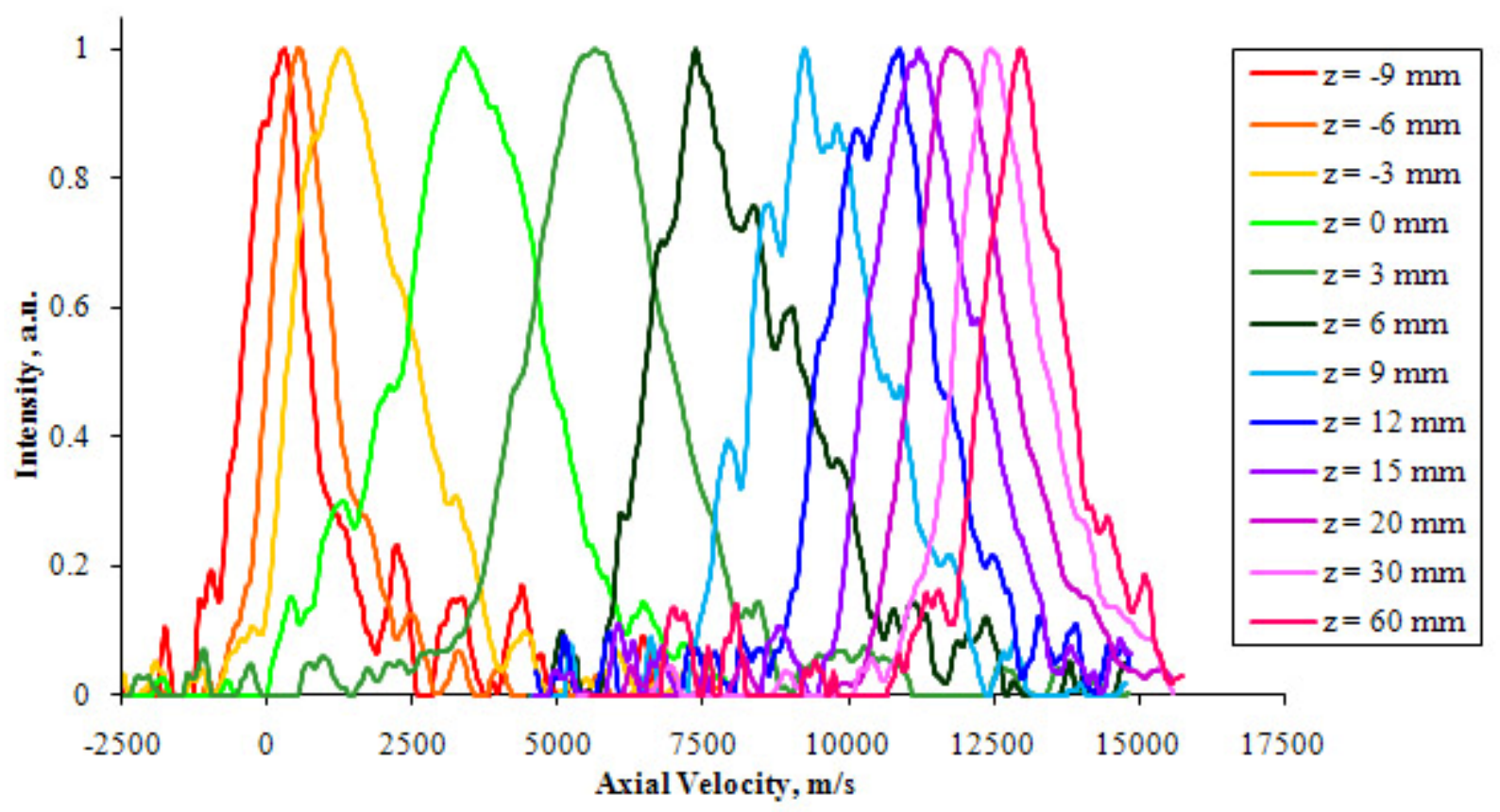

Figure 22. Evolution of velocity distribution functions for $150 \mathrm{~V}, 30 \mathrm{mg} / \mathrm{s} \mathrm{discharge.}$

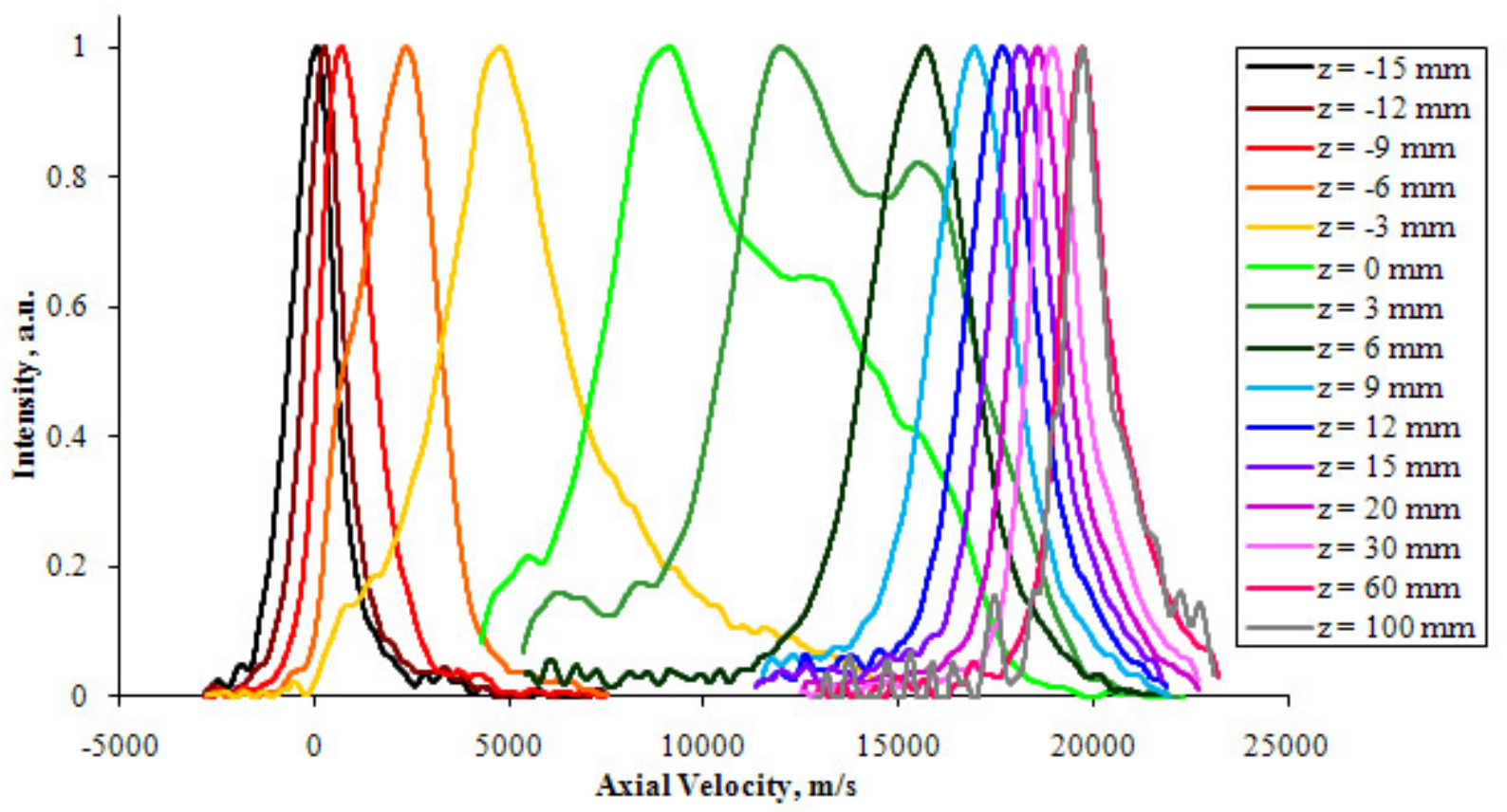

Figure 23. Evolution of velocity distribution functions for $300 \mathrm{~V}, 10 \mathrm{mg} / \mathrm{s}$ discharge. 


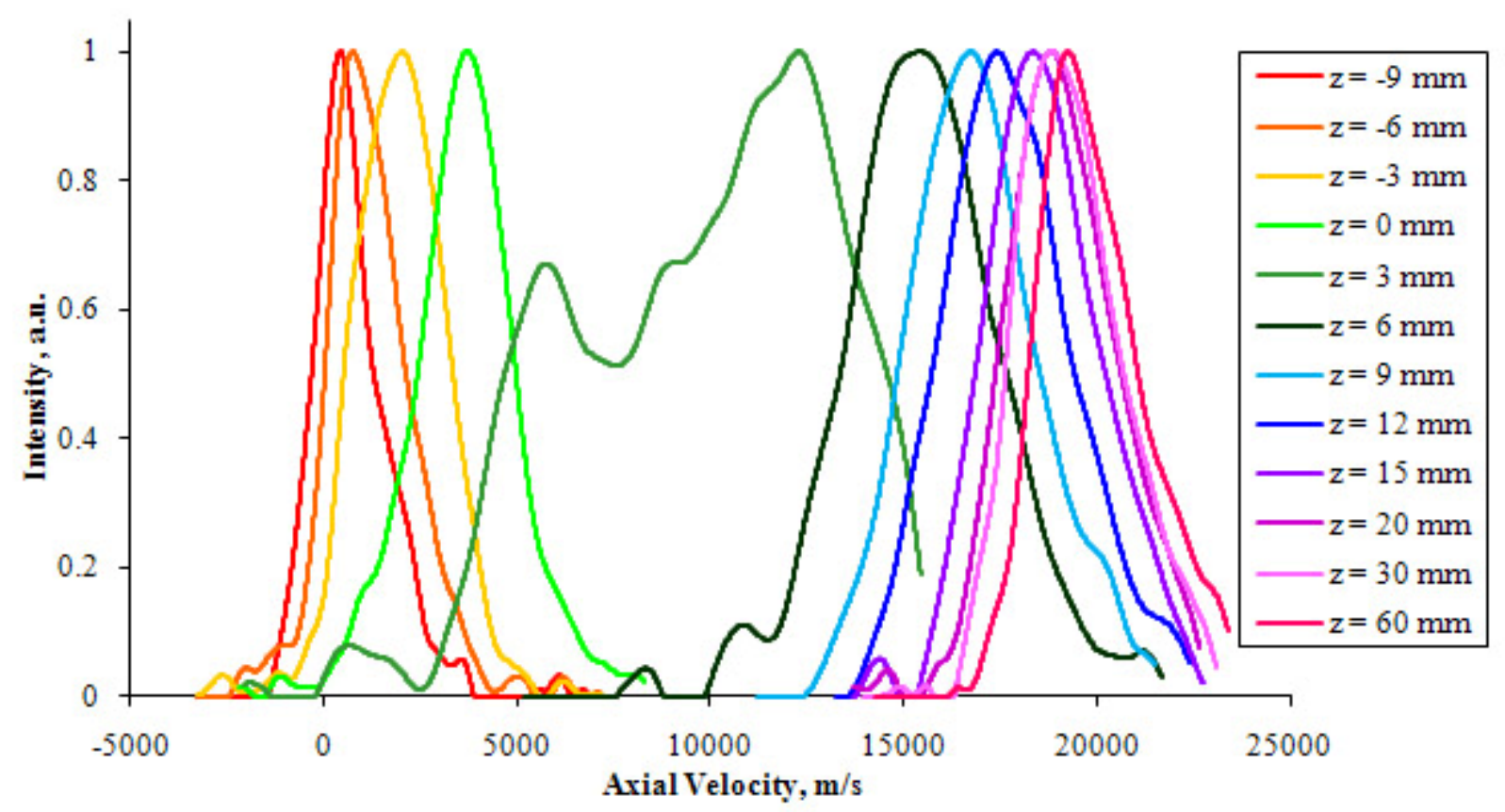

Figure 24. Evolution of velocity distribution functions for $300 \mathrm{~V}, 30 \mathrm{mg} / \mathrm{s}$ discharge.

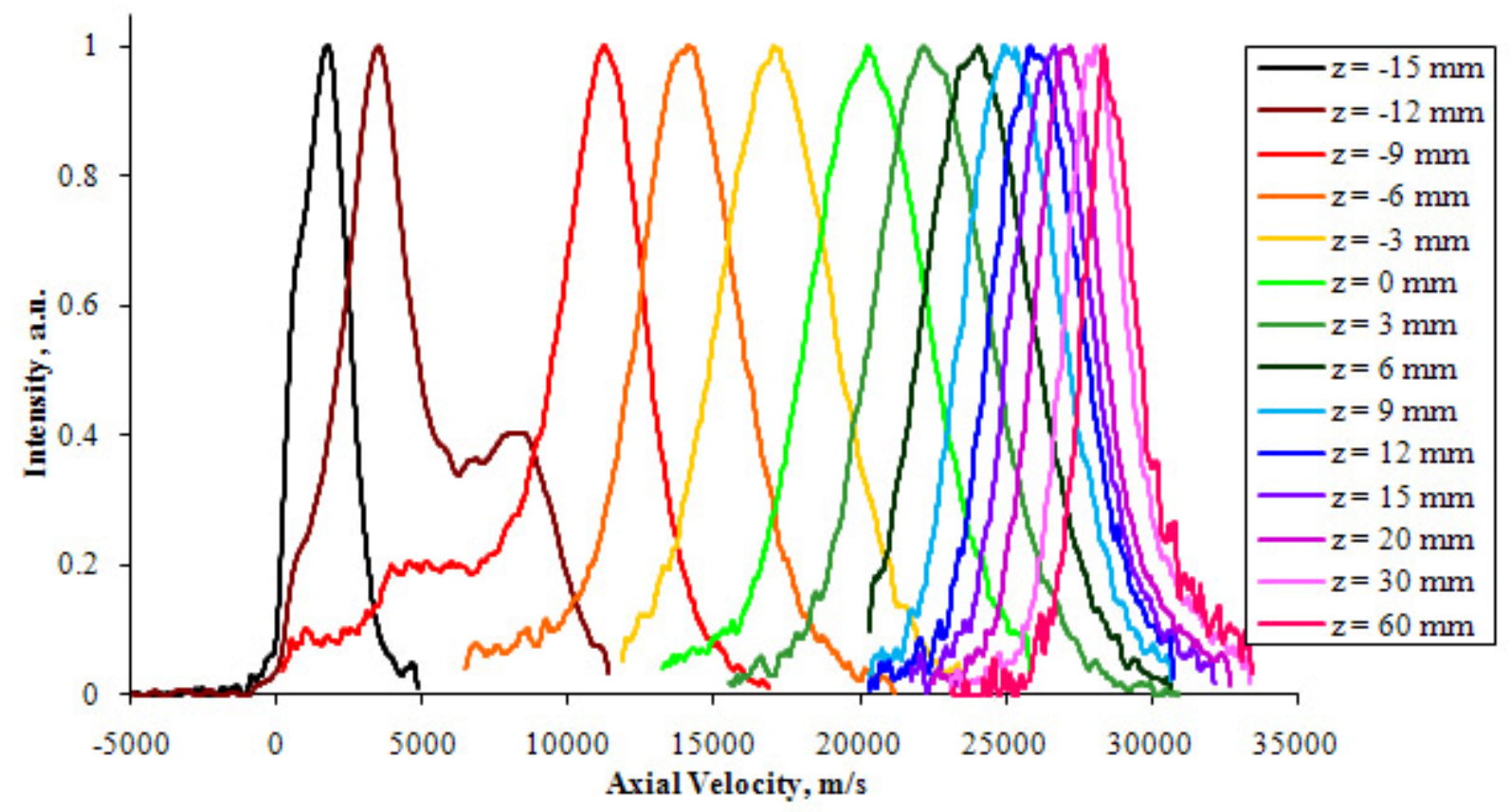

Figure 25. Evolution of velocity distribution functions for $600 \mathrm{~V}, 10 \mathrm{mg} / \mathrm{s} \mathrm{discharge.}$ 


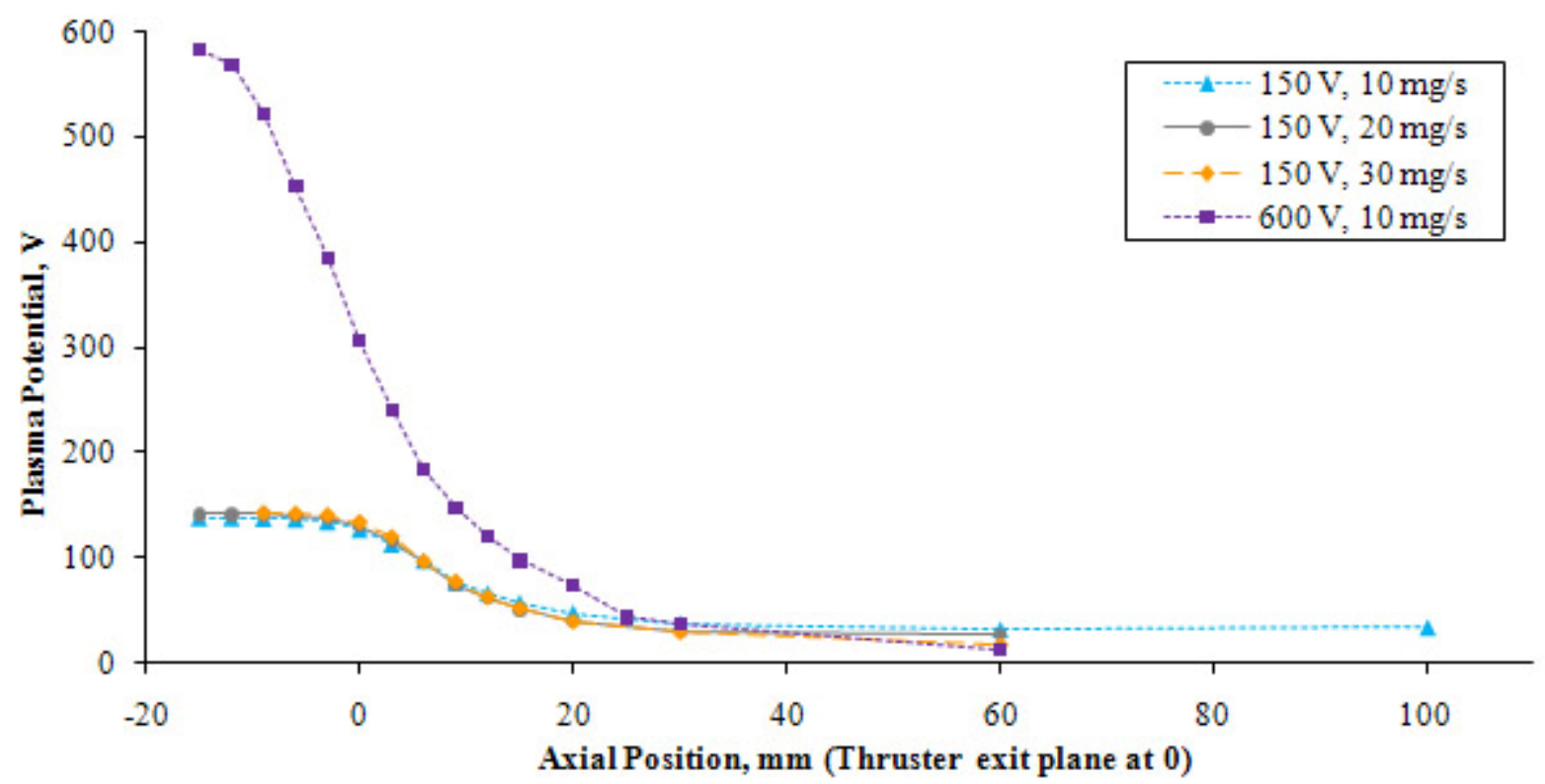

Figure 26. Calculated plasma potential for $150 \mathrm{~V}$ and $600 \mathrm{~V}$ operating conditions. 


\section{Acknowledgments}

The authors would like to acknowledge and thank the Air Force Office of Scientific Research (AFOSR) for funding this research. Dr. Mitat Birkan is the Project Manager for Grant FA9550-06-1-0105. The authors would also like to thank Dr. Tim Smith for his technical advice on laser-induced fluorescence.

\section{References}

${ }^{1}$ Lobbia, R. B. and Gallimore, A. D., "A Method of Measuring Transient Plume Properties", 44th AIAA/ASME/SAE/ASEE Joint Propulsion Conference \& Exhibit, AIAA-2008-4650, Hartford, CT, 21-23 Jul., 2008.

${ }^{2}$ Litvak, A. A., Raitses, Y., and Fisch, N. J., "Experimental Studies of High-Frequency Azimuthal Waves in Hall Thrusters", Physics of Plasmas, Vol. 11, No. 4, Apr., 2004, pp. 1701-1705.

${ }^{3}$ Choueiri, E. Y., "Plasma Oscillations in Hall Thrusters", Physics of Plasmas, Vol. 8, No. 4, Apr., 2001, pp. 1411-1426.

${ }^{4}$ Chesta, E., Lam, C. M., Meezan, N. B., Schmidt, D. P., and Cappelli, M. A., "A Characterization of Plasma Fluctuations within a Hall Discharge", IEEE Transactions on Plasma Science, Vol. 29, No. 4, Aug., 2001, pp. 582-591.

${ }^{5}$ Gascon, N., et al., "Signal Processing and Non-Linear Behavior of a Stationary Plasma Thruster: First Results", 35th AIAA/ASME/SAE/ASEE Joint Propulsion Conference \& Exhibit, AIAA-1999-2427, Los Angeles, CA, 20-24 Jun., 1999.

${ }^{6}$ Hargus, W. A., Jr. and Nakles, M. R., "Ion Velocity Measurements within the Acceleration Channel of a Lower Power Hall Thruster", 30th International Electric Propulsion Conference, 2007-172, Florence, Italy, 17-20 Sep., 2007.

${ }^{7}$ Nakles, M. R., Hargus, W. A., and Jr., "Background Pressure Effects on Internal and near-Field Ion Velocity Distribution of the Bht-600 Hall Thruster", 44th AIAA/ASME/SAE/ASEE Joint Propulsion Conference \& Exhibit, AIAA-2008-5101, Hartford, CT, 21-23 Jul., 2008.

${ }^{8}$ Hargus, W. A., Jr., Nakles, M. R., Tedrake, R., and Pote, B., "Effect of Anode Current Fluctuations on Ion Energy Distributions within a $600 \mathrm{~W}$ Hall Effect Thruster", 44th AIAA/ASME/SAE/ASEE Joint Propulsion Conference \& Exhibit, AIAA-2008-4724, Hartford, CT, 21-23 Jul., 2008.

${ }^{9}$ Smith, T. B., Ngom, B. B., Linnell, J. A., and Gallimore, A. D., "Diode Laser-Induced Fluorescence of Xenon Ion Velocity Distributions", 41st AIAA/ASME/SAE/ASEE Joint Propulsion Conference, AIAA-2005-4406, Tucson, AZ, 11-13 Jul., 2005.

${ }^{10}$ Gawron, D., Mazouffre, S., Sadeghi, N., and Heron, A., "Influence of Magnetic Field and Discharge Voltage on the Acceleration Layer Features in a Hall Effect Thruster", Plasma Sources Science and Technology, Vol. 17, $025001,2008$.

${ }^{11}$ Huang, W., Smith, T. B., and Gallimore, A. D., "Obtaining Velocity Distribution Using a Xenon Ion Line with Unknown Hyperfine Constants", 40th AIAA Plasmadynamics and Laser Conference, AIAA-2009-4226, San Antonio, Texas, 22-25 Jun., 2009.

${ }^{12}$ Svanberg, S., Atomic and Molecular Spectroscopy, $4^{\text {th }}$ ed., Springer-Verlag, Berlin, 2004.

${ }^{13}$ Harkness, H. W. and Heard, J. F., "The Stark Effect for Xenon", Proceedings of the Royal Society of London. Series A, Containing Papers of a Mathematical and Physical Character, Vol. 139, No. 838, 1 Feb., 1933, pp. 416-435.

${ }^{14}$ Smith, T. B., Huang, W., Ngom, B. B., and Gallimore, A. D., "Optogalvanic and Laser-Induced Fluorescence Spectroscopy of the Zeeman Effect in Xenon", 30th International Electric Propulsion Conference, 2007-229, Florence, Italy, 17-20 Sep., 2007.

${ }^{15}$ Hargus, W. A., Jr. and Charles, C. S., "Near Exit Plane Velocity Field of a 200-Watt Hall Thruster", Journal of Propulsion and Power, Vol. 24, No. 1, Jan.-Feb., 2008, pp. 127-133.

${ }^{16}$ Fife, J. M., Martinez-Sanchez, M., and Szabo, J., "A Numerical Study of Low-Frequency Discharge Oscillations in Hall Thrusters", 33rd AIAA/ASME/SAE/ASEE Joint Propulsion Conference, AIAA-1997-3052, Seattle, WA, 6-9 Jul., 1997.

${ }^{17}$ Boeuf, J. P. and Garrigues, L., "Low Frequency Oscillations in a Stationary Plasma Thruster", Journal of Applied Physics, Vol. 84, No. 7, 1 Oct., 1998, pp. 3541-3554.

${ }^{18}$ Petrov, G. M., "A Simple Algorithm for Spectral Line Deconvolution", Journal of Quantitative Spectroscopy \& Radiative Transfer, Vol. 72, No. 3, Feb., 2002, pp. 281-287.

${ }^{19}$ Reid, B. M. and Gallimore, A. D., "Plasma Potential Measurements in the Discharge Channel of a 6-Kw Hall Thruster", 44th AIAA/ASME/SAE/ASEE Joint Propulsion Conference \& Exhibit, AIAA-2008-5185, Hartford, CT, 21-23 Jul., 2008.

${ }^{20}$ Huang, W., Reid, B. M., Smith, T. B., and Gallimore, A. D., "Laser-Induced Fluorescence of Singly-Charged Xenon in a 6-Kw Hall Thruster Plume", 44th AIAA/ASME/SAE/ASEE Joint Propulsion Conference \& Exhibit, AIAA-2008-5102, Hartford, CT, 2123 Jul., 2008.

${ }^{21}$ Reid, B. M. and Gallimore, A. D., "Langmuir Probe Measurements in the Discharge Channel of a 6-Kw Hall Thruster", 44th AIAA/ASME/SAE/ASEE Joint Propulsion Conference \& Exhibit, AIAA-2008-4920, Hartford, CT, 21-23 Jul., 2008. 